\title{
Alteration of Polyketide Stereochemistry from anti to syn by a Ketoreductase Domain Exchange in a Type I Modular Polyketide Synthase Subunit
}

Clara H. Eng ${ }^{1,4}$, Satoshi Yuzawa ${ }^{3}$, George Wang ${ }^{5,6}$, Edward E. K. Baidoo ${ }^{5,6}$, Leonard Katz ${ }^{3,4}$, and Jay D. Keasling ${ }^{1,2,3,4,5,6}$

\footnotetext{
${ }^{1}$ Department of Chemical and Biomolecular Engineering, University of California, Berkeley, California 94270 , United States

${ }^{2}$ Department of Bioengineering, University of California, Berkeley, California 94270, United States

${ }^{3}$ QB3 Institute, University of California, Berkeley, California 94270, United States

${ }^{4}$ Synthetic Biology Engineering Research Center, 5885 Hollis Street, Emeryville, California 94608, United States

${ }^{5}$ Joint BioEnergy Institute, 5885 Hollis Street, Emeryville, California 94608, United States

${ }^{6}$ Biological Systems and Engineering Division, Lawrence Berkeley National Laboratory, Berkeley, California 94270, United States
} 


\section{Contents}

1 Experimental Procedures $\quad 3$

1.1 Authentic Synthetic Standards . . . . . . . . . . . . . . . . . . . . 3

1.2 Junction Design for KR Domain Exchanges . . . . . . . . . . . . . . . 3

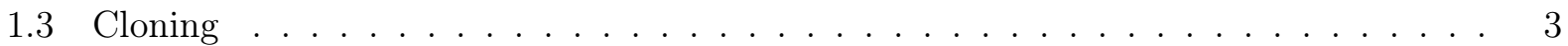

1.4 Protein Expression and Purification . . . . . . . . . . . . . . . . 3

1.5 In Vitro Production of 3a-d by LipPks1+TE Variants . . . . . . . . . . . . . . . . 4

1.6 In Vitro Production of $\mathbf{2 a - d}$ by LipPks1+TE Variants . . . . . . . . . . . . . . . . . 4

1.7 Stereochemical Purity of Enzymatically Produced $\mathbf{3 a} \ldots \ldots \ldots$. . . . . . . . . 5

2 Schematic of Ketoreductase Domain Exchange Junctions in LipPks1+TE 6

$\begin{array}{lll}3 & \text { Properties of Donor Ketoreductase Domains } & 7\end{array}$

4 Sequence Alignment of Donor Ketoreductase Domains $\quad 8$

5 Gene Synthesis $\quad 10$

6 Primers $\quad 14$

$\begin{array}{llr}7 & \text { Plasmids } & 16\end{array}$

8 Soluble Expression of (-DE)LipPks1+TE (SDS-PAGE) 17

9 Purity of LipPks1+TE Variants (SDS-PAGE) 18

10 Production of 3a-d by LipPks1+TE Variants (LC-MS Chromatograms) 19

11 Production of 2a-d by LipPks1+TE Variants (LC-MS Chromatograms) 24

12 Stereochemical Purity of 3a Produced by A-type LipPks1+TE Variants (Chiral GC-MS Chromatograms) $\quad 29$

13 Activity of LipPks1+TE Variants Using 1d as a Starter Substrate 38

14 Relative Production of 3a-d and 2a-d by LipPks1+TE Variants 39 


\section{Experimental Procedures}

\subsection{Authentic Synthetic Standards}

Racemic mixtures of syn 3a-d were synthesized as previously described. ${ }^{14}$ (2R,3S)-3a (syn A1 type product) and $(2 S, 3 S)-\mathbf{3 a}$ (anti A2 type product) were purchased from Organic Consultants Incorporated. All other chemicals were purchased from Sigma-Aldrich unless otherwise described.

\subsection{Junction Design for KR Domain Exchanges}

All unique accession numbers associated with type I modular PKS KS domains present in the curated Clustermine360 database ${ }^{3}$ were compiled into a local BLAST nucleotide database using BLAST $+{ }^{2}$ The 45 homologs of LipPks1 present in this local database which had over $75 \%$ sequence coverage of the query were identified using tblastn. Next, the mafft-linsi algorithm from the Mafft software package ${ }^{8}$ was used to perform a sequence alignment and provide an estimate of sequence conservation. The amino acid sequences corresponding to the KR and ACP structures present in the Protein Data Bank (PDB) ${ }^{1,9,10,16-18}$ were separately aligned with the amino acid sequence of LipPks1, again using the mafft-linsi algorithm. These two sequence alignments were used to select junctions for KR domain exchanges.

\subsection{Cloning}

A LipPks1+TE variant in which the DE was removed was generated by PCR amplication followed by phosphorylation and ligation. This DE deletion variant differed from wild-type LipPks1+TE only in that a 54 amino acid sequence preceding the KR starting with RPA and ending with AAV, shown in Supplementary Figure 1, was removed. A nine amino acid spacer sequence between the AT and $\mathrm{KR}$ was left intact, consistent with what is typically observed in modules lacking a native DE. Gene fragments encoding Amp $\mathrm{DE}_{2}, \mathrm{Amp} \mathrm{KR}_{2}$, Con $\mathrm{DE}_{2}$, and Con $\mathrm{KR}_{2}$ were purchased as gBlocks from Integrated DNA Technologies. E. coli codon optimized synthetic genes encoding additional donor KR domains were ordered either from Genewiz (Amp KR 1 , Pik $\mathrm{KR}_{1}$, and Spn $\mathrm{KR}_{3}$ ) or from Genscript (Ave $\mathrm{KR}_{1}$, Ery $\mathrm{KR}_{1}$, and Ery $\mathrm{KR}_{6}$ ). Bor $\mathrm{KR}_{1}$ was amplified from the plasmid pARH100. ${ }^{6}$ All cloning was performed in the E. coli strain DH10B. Plasmid backbones containing LipPks1+TE but lacking either the native $\mathrm{DE}$ and $\mathrm{KR}$, or just the native $\mathrm{KR}$ were amplified from pSY044. ${ }^{14}$ All oligonucleotides used as primers in this study were designed using j5 DNA assembly design software, ${ }^{7}$ and are shown in Supplementary Tables 2 and 3. Plasmids were constructed using a Golden Gate protocol adapted from work by Engler et. al. ${ }^{4,5}$ for use with j5. ${ }^{7}$

\subsection{Protein Expression and Purification}

Expression vectors containing LipPks1+TE variants were transformed into the E. coli strain K2073. ${ }^{11}$ Cultures were grown at $37^{\circ} \mathrm{C}$ in Luria-Bertani (LB) medium supplemented with the appropriate antibiotic until an $\mathrm{OD}_{600}$ of $0.35-0.45$ was reached. The cultures were cooled to $18^{\circ} \mathrm{C}$, induced with $250 \mu \mathrm{M}$ isopropyl- $\beta$-D-galactopyranoside (IPTG), and further incubated at $18^{\circ} \mathrm{C}$ for $18 \mathrm{~h}$. Cells were collected by centrifugation ( $5000 \mathrm{rpm}, 5 \mathrm{~min}$, room temperature), and resuspended in $50 \mathrm{~mL}$ suspension buffer (50 mM phosphate buffer, $\mathrm{pH}$ 7.6, $300 \mathrm{mM}$ sodium chloride, $10 \mathrm{mM}$ imidazole)

per $1 \mathrm{~L}$ culture. The cells were lysed by sonication, and cellular debris removed by three consecutive centrifugation steps $\left(8000 \mathrm{rpm}, 15 \mathrm{~min}, 4^{\circ} \mathrm{C}\right)$. The supernatant was mixed with $10 \mathrm{~mL}$ Nickel-NTA 
agarose resin (Qiagen) per $1 \mathrm{~L}$ culture for $1 \mathrm{~h}$ at $4^{\circ} \mathrm{C}$. The resulting mixture was added to a fritted column, washed with 18 resin volumes of suspension buffer, and the protein eluted with four resin volumes of elution buffer (150 mM phosphate buffer, $\mathrm{pH} 8.3,50 \mathrm{mM}$ sodium chloride, $150 \mathrm{mM}$ imidazole). The eluted protein was further purified by anion exchange using a HiTRAP Q column (GE Healthcare). A mixture of $50 \mathrm{mM}$ phosphate buffer, $\mathrm{pH} 7.6,8 \% \mathrm{w} / \mathrm{v}$ glycerol (buffer A) and 50 $\mathrm{mM}$ phosphate buffer, $\mathrm{pH} 7.1,500 \mathrm{mM}$ sodium chloride, $8 \% \mathrm{w} / \mathrm{v}$ glycerol (buffer B) was used as the mobile phase, and the protein purified using the following gradient method: 0 to $40 \%$ B over 5 min at a flow rate of $2.5 \mathrm{ml} / \mathrm{min}$, hold at $40 \% \mathrm{~B}$ for $10 \mathrm{~min}$ using a flow rate of $1 \mathrm{~mL} / \mathrm{min}, 40$ to $100 \%$ $\mathrm{B}$ over $40 \mathrm{~min}$ at a flow rate of $1 \mathrm{~mL} / \mathrm{min}$. The purified protein was concentrated using a Amicon Ultra-15 centrifugal filter unit with Ultracel-100 membrane (Millipore) and stored at $-80^{\circ} \mathrm{C}$.

\subsection{In Vitro Production of 3a-d by LipPks1+TE Variants}

Each LipPks1+TE variant $(0.5 \mu \mathrm{M})$ was incubated in a $50 \mu \mathrm{L}$ sample containing $100 \mathrm{mM}$ sodium phosphate buffer (pH 7.2), $2.5 \mathrm{mM}$ TCEP, $500 \mu \mathrm{M}$ NADPH, $216 \mu \mathrm{M}$ starter acyl-CoA (one of 1a-d), and $236 \mu \mathrm{M}$ methylmalonyl-CoA. After incubation at room temperature for $17.5 \mathrm{~h}$, samples were quenched using $50 \mu \mathrm{L}$ methanol. ${ }^{14}$

Separation by $\mathrm{LC}$ was performed at $50^{\circ} \mathrm{C}$ on an Agilent 1100 series high-performance liquid chromatography system (Agilent Technologies) using an Inertsil ODS-3 reverse-phase column (250 $\mathrm{mm}$ length, $2.1 \mathrm{~mm}$ internal diameter, $3 \mu \mathrm{m}$ particle size; General Life Sciences). A mixture of $0.1 \%$ formic acid in water (solvent $\mathrm{A}$ ) and $0.1 \%$ formic acid in methanol (solvent B) was used as the mobile phase, and the products were separated using the following gradient method: 60 to $100 \% \mathrm{~B}$ over $10 \mathrm{~min}$, hold at $100 \% \mathrm{~B}$ for $2 \mathrm{~min}, 100$ to $60 \%$ B over $1 \mathrm{~min}$, hold at $60 \%$ B for $17 \mathrm{~min}$, all at a constant flow rate of $0.13 \mathrm{~mL} / \mathrm{min}$. A sample injection volume of $10 \mu \mathrm{L}$ was used.

The LC system was coupled to an Agilent 1100 series mass spectrometer detector (Agilent Technologies). Atmospheric pressure ionization electrospray (API-ES) was used to produce gasphase ions. Nitrogen gas at a flow rate of $10 \mathrm{~L} / \mathrm{min}$ and pressure of $30 \mathrm{lb} / \mathrm{in}^{2}$ was used for both nebulization and drying. A drying gas temperature of $330^{\circ} \mathrm{C}$ was used. Electrospray ionization (ESI) was performed in negative ion mode with a capillary voltage of $3.5 \mathrm{kV}$. The instrument was tuned for a $\mathrm{m} / z$ range of 50 to 3000 using the Agilent ESI tuning mix. $[\mathrm{M}-\mathrm{H}]^{-}$ions were detected in selected ion monitoring (SIM) mode $(\mathbf{3 a}, \mathrm{m} / z$ 145; 3b, $\mathrm{m} / z$ 131; 3c, $\mathrm{m} / z$ 145; 3d, $\mathrm{m} / z$ 159 ) with a fragmentor voltage of $70 \mathrm{~V}$, gain of $5 \mathrm{EMV}$, and actual dwell of 746 . The ionization efficiencies of stereoisomers were assumed to be identical. Data acquisition was performed using ChemStation software (Agilent Technologies), and data analysis was performed using MassHunter software (Agilent Technologies).

\subsection{In Vitro Production of 2a-d by LipPks1+TE Variants}

Each LipPks1+TE variant $(0.5 \mu \mathrm{M})$ was incubated in a $50 \mu \mathrm{L}$ sample containing $100 \mathrm{mM}$ sodium phosphate buffer (pH 7.2), $2.5 \mathrm{mM}$ TCEP, $216 \mu \mathrm{M}$ starter acyl-CoA (one of 1a-d), and $236 \mu \mathrm{M}$ methylmalonyl-CoA in water. After incubation at room temperature for $17.5 \mathrm{~h}$, samples were quenched using $50 \mu \mathrm{L}$ methanol. The samples were then sealed, incubated at $50^{\circ} \mathrm{C}$ overnight, and subsequently chilled at $4^{\circ} \mathrm{C}$ for 1 hour to obtain ketone products. ${ }^{15}$

Separation by $\mathrm{LC}$ was conducted at $55^{\circ} \mathrm{C}$ with a Kinetex XB-C18 reversed phase column (100 mm length, $2.1 \mathrm{~mm}$ internal diameter, $2.6 \mu \mathrm{m}$ particle size; Phenomenex) using an Agilent 1200 Rapid Resolution LC system (Agilent Technologies). The mobile phase was composed of water 
(solvent A) and methanol (solvent B). $\mathbf{2} \mathbf{a}-\mathbf{b}$ and $\mathbf{2 d}$ were each separated via the following gradient: 25 to $100 \%$ B over $1.5 \mathrm{~min}$, hold at $100 \%$ B for $3 \mathrm{~min}, 100$ to $25 \%$ B over $0.2 \mathrm{~min}$, and hold at $25 \%$ B for an additional $5.3 \mathrm{~min}$. 2c was separated via the following gradient: 50 to $100 \%$ B over 1.5 min, hold at $100 \%$ B for $3 \mathrm{~min}, 100$ to $50 \%$ B over $0.2 \mathrm{~min}$, and hold at $50 \%$ B for an additional 5.3 min. For both separation methods, the sample injection volume was $10 \mu \mathrm{L}$, and the following flow rate program was used: hold constant at $0.24 \mathrm{~mL} / \mathrm{min}$ from 0 to $4.5 \mathrm{~min}$, increase from 0.24 to 0.4 $\mathrm{mL} / \mathrm{min}$ over $0.2 \mathrm{~min}$, and hold constant at $0.4 \mathrm{~mL} / \mathrm{min}$ for an additional $5.3 \mathrm{~min}$. This resulted in a total run time of $10 \mathrm{~min}$ for each method.

The Agilent 1200 Rapid Resolution LC system was coupled to an Agilent 6210 time-of-flight (TOF) mass spectrometer (Agilent Technologies). Nitrogen gas was used as both the nebulizing and drying gas to facilitate the production of gas-phase ions. The drying and nebulizing gases were set to $10 \mathrm{~L} / \mathrm{min}$ and $30 \mathrm{lb} / \mathrm{in}^{2}$, respectively, and a drying gas temperature of $320^{\circ} \mathrm{C}$ was used throughout. Atmospheric pressure chemical ionization (APCI) was conducted in the positive-ion mode with capillary and fragmentor voltages of $3.5 \mathrm{kV}$ and $100 \mathrm{~V}$, respectively. The skimmer, OCT1 $\mathrm{RF}$, and corona needle were set to $50 \mathrm{~V}, 170 \mathrm{~V}$, and $4 \mu \mathrm{A}$, respectively. The vaporizer was set to $350^{\circ} \mathrm{C}$. 2a-d were detected via $[\mathrm{M}+\mathrm{H}]^{+}$ions: $\mathbf{2 a}, \mathrm{m} / z=101.09609 ; \mathbf{2 b}, \mathrm{m} / z=87.08044 ; \mathbf{2 c}, \mathrm{m} / z$ $=101.09609 ; \mathbf{2 d}, \mathrm{m} / z=115.11174$. The analysis was performed using an $\mathrm{m} / z$ range of 66 to 166 . Data acquisition and processing were performed using MassHunter software (Agilent Technologies).

\subsection{Stereochemical Purity of Enzymatically Produced 3a}

GC-MS was performed on an Agilent 6890 gas chromatography system (Agilent Technologies) equipped with an Agilent 5973 mass selective detector (Agilent Technologies). $1 \mu \mathrm{L}$ samples were injected onto a CycloSil-B chiral capillary column (30 m length, $0.25 \mathrm{~mm}$ internal diameter, 0.25 $\mu \mathrm{m}$ film thickness; Agilent Technologies) and GC separation was carried out with the following oven temperature program, following a previously reported ${ }^{13}$ method: hold at $50^{\circ} \mathrm{C}$ for $2 \mathrm{~min}$, ramp up to $90^{\circ} \mathrm{C}$ at a rate of $1^{\circ} \mathrm{C} / \mathrm{min}$, then ramp up to a final temperature of $200^{\circ} \mathrm{C}$ at a rate of $20^{\circ} \mathrm{C} / \mathrm{min}$. 3a was detected by monitoring the fragment ions $m / z$ 117, 88, and 45 in SIM mode. ChemStation software (Agilent Technologies) was used for data acquisition and analysis. 


\section{Schematic of Ketoreductase Domain Exchange Junctions in LipPks1+TE}

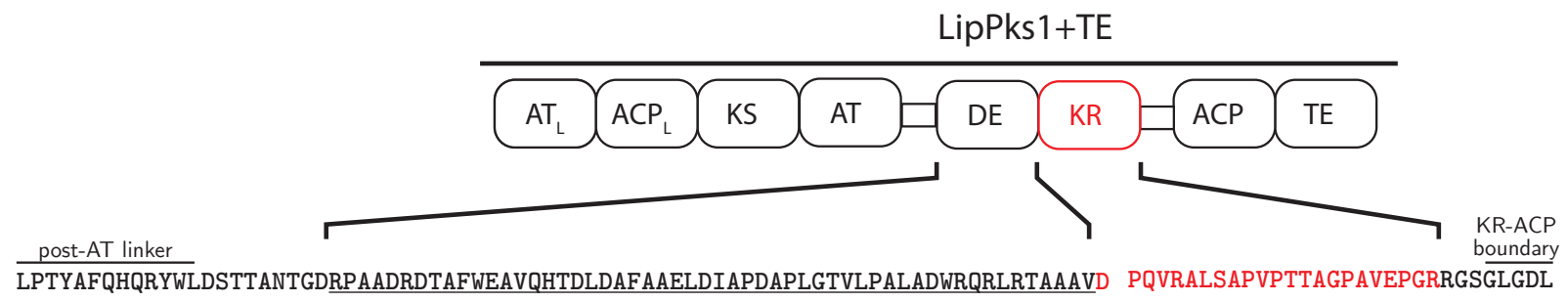

Figure 1: KR domain exchange junctions selected in LipPks1+TE with the DE underlined, and the KR shown in red. The conserved post-AT linker ${ }^{12}$ and the conserved motif that marks the beginning of the $\mathrm{ACP}^{1}$ are also annotated. 


\section{Properties of Donor Ketoreductase Domains}

Table 1: Properties of donor ketoreductase domains.

\begin{tabular}{|c|c|c|c|c|}
\hline $\mathrm{KR}$ & Type & $\mathrm{DE}$ & Length (a.a.) & Product in native PKS \\
\hline Lip $\mathrm{KR}_{1}$ & A2 & + & 499 & \\
\hline Amp $\mathrm{KR}_{1}$ & $\mathrm{~A} 2$ & + & 483 & \\
\hline Amp $\mathrm{KR}_{2}$ & A1 & + & 488 & \\
\hline Ave $\mathrm{KR}_{1}$ & B1 & + & 488 & \\
\hline Bor $\mathrm{KR}_{1}$ & B & - & 496 & \\
\hline Con $\mathrm{KR}_{2}$ & B1 & + & 481 & \\
\hline Ery $\mathrm{KR}_{1}$ & B2 & - & 474 & \\
\hline Ery $\mathrm{KR}_{6}$ & A1 & - & 437 & \\
\hline Pik KR 1 & $\mathrm{~B} 2$ & - & 481 & \\
\hline Spn $K_{3}$ & A1 & + & 480 & \\
\hline
\end{tabular}




\section{Sequence Alignment of Donor Ketoreductase Domains}

LipKR1

AmpKR1

AmpKR2

AveKR1

BorkR1

ConKR2

EryKR1

EryKR6

PikKR1

SpnKR3

LipKR1

AmpKR1

AmpKR2

AveKR1

BorkR1

ConKR2

EryKR1

ErYKR6

PikKR1

SpnKR3

LipKR1

AmpKR1

AmpKR2

AveKR1

BorkR1

ConKR2

EryKR1

ErYKR6

PikKR1

SpnKR3

LipkR1

AmpKR1

AmpKR2

AveKR1

BorkR1

ConKR2

EryKR1

EryKR6

PikKR1

SpnKR3

LipKR1

AmpKR1

AmpKR2

AveKR1

BorkR1

ConKR2

EryKR1

EryKR6

PikKR1

SpnKR3

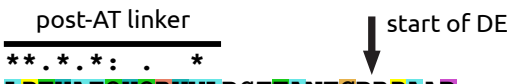

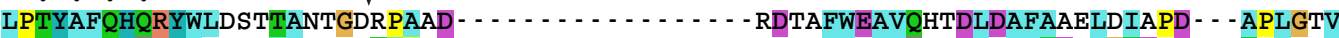

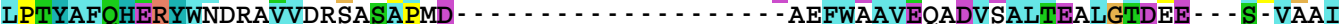

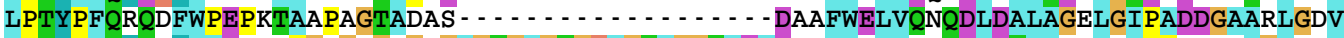
LPTYPFQHQHYWLESSQPGAGSGSGAGAGSGAGSGRAGTAGGTAEVESRFWDAVARQDLETVATTLAVPPS - - - AGLDTV LPTYPFQHQHYWLDVPPLFTASSAAQD $\ldots \ldots \ldots$ LPTYPFQRRRYWLEAPRASDSAAPADD - - . - . - - - - - - - GLWREIEAQDSAALADTLGVDPG - - A - VGEV

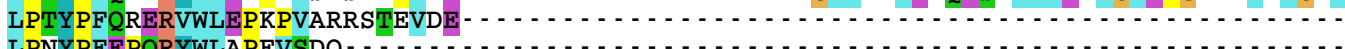
LPNYPFEPQRYWLAPEVSDQ - _ _ $\ldots \ldots \ldots$ LPTYAFOAERYWLENTPAAL $\cdots \cdots$

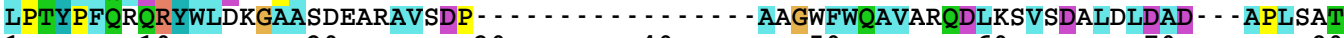

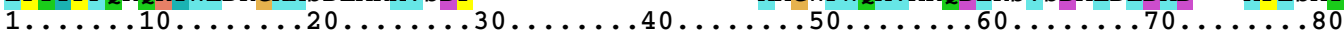
end of DE $\mid$ start of KR

LPALADWRQRLRTAAAVDAWRYRTAFKRLPDAPGAPV - - LTGSWLAVVPVRHLDDPSVTTSLDAVAKAGAEVVQLAIEDA LPGLASWRRTRGERSTVDSWRYRVVWEPLAQIPRAT - - - LDGTWLLVSAGGVDDD - - - - VAEVLEAGGAEVRRLVL - DE LPALSAWRTRSLTRSRADALRYHIEWNRVAEPGTAR - - - PAGRLLAVISPDHAGAPWVTAVLDAL - - - GPDTVRFEA - - K VPALSAWHRHQHDQARINTWTYQETWKPLTLPTTHQ - - - PHQTWLIAIPETQTHHPHITNILTNLHHHGITPIPLTL - NH - - - - - - - - - - - GGWRYR IHWRRLGTRDSGDR - - LSGRWLLLVPESDGTEPWVEGAEKMLAERGCEVVHVPI - AA VPALSAWRSERIRGRRADSWRHRLGWRPLTLAPRAS - - LSGRWLVVLPATDSG - - - ADVLAALTAAGAEPVPVVVSAP - . - . - . - - - - VSALRYRIEWRPTGAGEPAR - - - LDGTWLVAKYAGTADET - STAAREALESAGARVRELVV - DA - . - . - . - . - . - LADSRYRVDWRPLATTPVD - - - LEGGFLV - - - - HGSAP - - ESLTSAVEKAGGRVVPVAS - - - - . - - - - - ATGDDWRYRIDWKRLPAAEGSERTGLSGRWLAVTPEDHSAQA - - AAVLTALVDAGAKVEVLTA - GA LPALSVWHRQERERVLADGWRYRVDWVRVAPQPVRR - - TRETWLLVVPPGGIEEALVERLTDALNTRGISTLRLDV - PP

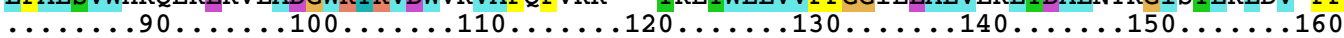

$:$
DADVDR - LTERLRGLVAG - - - LGAAPAGIMSFLGLDEERHRDHPAMPSGLATSLALVRALGRAGIGAPLWMVTREAVAA TCTDPAVLREHLTDT - - - . - - - DGLTGIVSVLADAEEGCVRHPGLTLGLALSVSLAQALGEADVTAPLWFLTCGAFST GTDRAA - WAAQLAQLRED - - - - EGEFHAVVSLLAAAEALHTDHGSVPLGLAQTLLLAQALGDAGLTAPLWCLTRGGVAA THTNPQHLHHTLHHTRQQAQNHTTGAITGLLSLLALDETPHPHHPHTPTGTLLNLTLTQTHTQTHPPTPLWYATTNATTT TADRDA - MVGAVRESVED - - - - GRVDGVLSLLALDGRPHPDAAAVPTGLVATAQVVQVSDELGI - GPLWVATRQAVSV DQDRES - LATSLRAASGA - - - - - EPVAGVFSLLAEETHPHPAQPDVPAGLSLTLALVQALGDTGTDAPLWCATRALAAV RCGRDE - LAERLRSV - - - - - - - - GEVAGVLSLLAVDEAEPEEAPLALASLADTLSLVQAMVSAELGCPLWTVTESAVAT - ADREA - LAAALREV - - - - - - PGEVAGVLS - - - - - - - - - VHTGAATHLALHQSLGEAGVRAPLWLVTSRAVAL DDDREA - LAARLTALTTG - - . - DGFTGVVSLL - . . . . - . - . - DGLVPQVAWVQALGDAGIKAPLWSVTQGAVSV AATSGE - LATELRAAADG - - - - - DPVKAILSLTALDERPHPECKDVPSGIALLLNLVKALGEADLRI PLWTITRGAVKA

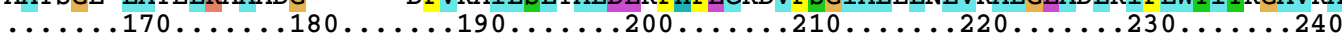

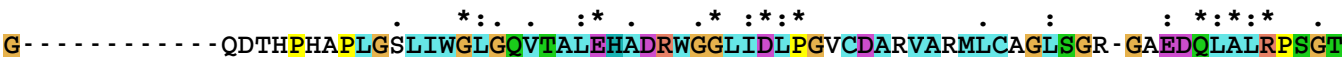
G - . - . - - - - PSDTVTRPLQSQIAGLGWTVAVEHPHRWGGGVDLPEALDARAAQRLVAALAGALGDDDQLAVRPAGV G - - - - - - - RGDVLSSPVQGALWGLGRVIGLEHPDRWGGLIDLPETVDTRAAARLTGLLADA - GGEDQLAIRGSGV H - - . - . - - - PNDPLTHPTQAQTWGLARTTLLEHPTHTAGIIDLPTTPTPHTLQHLTQTLTQP - HHQTQLAIRTTGT DGADEADGAGRTRKADDPADVAQAAVWGLGRVAALEKPRLWGGLVDLPARADERMRDLVAQALTAP - DAEDQLAVRADGI G - - - - - - GGERPGAGAQAAVAGLGRTVALERPDTWGGLVDLPAVLDERAADRLGAVLATV - TDEDQVAVRSSGV G - - . - . - - - PFERVRNAAHGALWGVGRVIALENPAVWGGLVDVPAGSVAELARHLAAVVSGG - AGEDQLALRADGV G - - - - - - - - ESE - PVDPEQAMVWGLGRVMGLETPERWGGLVDLPAEPAPGDGEAFVACLGAD - GHEDQVAIRDHAR

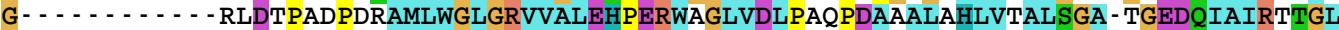
G - . . . . - . PADRLLRPMQAQAWGLGRVAALEHPERWGGLIDLPDSLDGDVLTRLGEALTNG - LAEDQLAIRQSGV $\ldots \ldots 250 \ldots \ldots 260 \ldots \ldots 270 \ldots \ldots 280 \ldots \ldots 290 \ldots \ldots 30 \ldots \ldots \ldots$

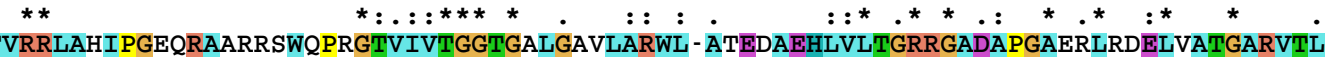
LARRIVRASGDTRRKARSWKPRGTTLVTGGSGTLAPGLARHL - AAQGAEHLVLLSRRGADAPGAAELAAELQAAGTEVRF LARRLAHAAPAVPGSGKRP PVHGSVLVTGGTGGIGGRVARRL - AEQGAAHLVLTSRRGADAPGAAELRAELEQLGVRVTI HTRRLTPTTLTPTHQPPTPTPHGTTLITGGTGALATHLTHHLTTHQPTQHLLLTSRTGPHTPHAQHLTTQLQQKGIHLTI AVRRLVRSAAS - - APADDWQPSGTVLVTGGTGGVGANVARWL - VTQDIQHLLLVSRRGPDAPGAAELLAELSASGTSVTI YGRRLLPVGPG - RPAEARHSP SGTVLITGGTGGTGAQVAREL - AERGAAHLLLLSRRGPDAPGADELRAELAALGARVTV YGRRWVRAAAP - - ATDDEWKPTGTVLVTGGTGGVGGQIARWL - ARRGAPHLLLVSRSGPDADGAGELVAELEALGARTTV YGRRLVRAPLGTRES - - SWEPAGTALVTGGTGALGGHVARHL - ARCGVEDLVLVSRRGVDAPGAAELEAELVALGAKTTI HARRLARAPLHGRRPTRDWQPHGTVLITGGTGALGSHAARWM - AHHGAEHLLLVSRSGEQAPGATQLTAELTASGARVTI LARRLVPAPAN - QPAGRKWRPRGSALITGGLGAVGAQVARWL - AEIGAERIVLTSRRGNQAAGAAELEAELRALGAQVSI $\ldots \ldots \ldots 330 \ldots \ldots 340 \ldots \ldots 350 \ldots \ldots .360 \ldots \ldots 370 \ldots \ldots 380 \ldots \ldots 0 \ldots$ 
LipKR1

AmpKR1

AmpKR2

AveKR1

BorkR1

ConKR2

EryKR1

EryKR6

PikKR1

SpnKR3

LipKR1 AmpKR1

AmpKR2

AveKR1

BorKR1

ConKR2

EryKR1

ErykR6

PikKR1

SpnKR3

LipKR1

AmpKR 1

AmpKR2

AveKR1

BorKR 1

ConKR2

ErykR1

ErykR6

PikKR1

SpnKR3

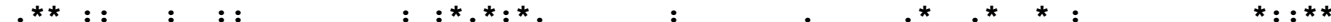
AACDVADRKAVAALLDELAADGETVRAVLHAAGVA-DLTSLENTGPEAFAAGVAAKVDGALHLTELLDHDSLDAFVLFSS AACDITDPDAVAALLADLKAEGRTVRTVVHTAAVI - ELAALADTTVDAFADVVHAKVTGARILDELLDDEELDDFVLYSS AACDAADREALAALLAELPED - APLTAVFHSAGVAHDDAPVADLTLGQLDALMRAKLTAARHLHELTADLDLDAFVLFSS TTCDTSNPDQLQQLLNTI P PQ - HPLTTVIHTAGIL - DDATLTNLTPTQLNNVLRAKAHSAHLLHQLTQHTPLTAFVLYSS EPCDVTDADAVRRLIGAVPAE - RPLSTVVHAAGVL - DDCLIDALTPQRLAAALEVKAKGALNLHEAAGEAHL - - - VLFSS AACDVADRAQLAAVVAAI PEE - EPLTTVVHAAGVL - DDGTLDALDPARLATLMRVKVTAARTLHEVTDGIGLADFVVFSS AACDVTDRESVRELLGGIGDD - VPLSAVFHAAATL - DDGTVDTLTGERIERASRAKVLGARNLHELTRELDLTAFVLFSS TACDVADREQLSKLLEELRGQGRPVRTVVHTAGVP - ESRPLHEIG - - ELESVCAAKVTGARLLDELCP - - DAETFVLFSS AACDVADPHAMRTLLDAIPAE - TPLTAVVHTAGAL - DDGIVDTLTAEQVRRAHRAKAVGASVLDELTRDLDLDAFVLFSS VACDVTDRAEMSALLAE - - - FDVTAVFHAAGVG - RLLPLAETDQNGLAEICAAKVRGAQVLDELCDSTDLDAFVLFSS $\ldots \ldots 410 \ldots \ldots 420 \ldots \ldots 430 \ldots \ldots 440 \ldots \ldots 450 \ldots \ldots 460 \ldots \ldots 470 \ldots \ldots 40$

** * * $::: \quad:: * * *$ IAGVWGSGDHGAYAAANAFLNALAEYNRARGIPTTSIAWGVWNAFGVEGAGGISEAVDLDQLHRRGLPLIEPELGLTALR TAGMWGSGVHAAYVAGNAYLSALAEQRRARGARATS IHWGKWPDDLE - - - - - - RELADPHQIRRSGLEYLDPELAMTALT GAAVWGSGGOPGYAAANAYLDALAEHRRSLGLTASSVAWGTWGEVGMA - - - - - - - TDPEVHDRLVROGVLAMEPEHALGALD AAATFGAPGQANYAAANAYLDALAHHRHTHHLPATS IAWGTWQGNGLA - - - - - - DSDKARAYLDRRGFRPMS PELATAAVT LAGTTGTKGQGNYAAANAYLDALAERRRADGLPATSVAWGAWQGAGMV - - - - - - ADAAVAHRTRRYGLPLMSPDRAVATLR FMGVLGSAGOGNYAAANAALDALVAERRAAGLPGTSVAWGAWAGGGM - - - - - - - - - VDDRIAERLRRLGITPMEPRAAVRAMT FASAFGAPGLGGYAPGNAYLDGLAQQRRSDGLPATAVAWGTWAGSGM - - - - - - - - AEPAVADRFRRHGVIEMPPETACRALQ GAGVWGSANLGAYSAANAYLDALAHRRRAEGRAATSVAWGAWAGEGM - - - - - - - ATGDL - EGLTRRGLRPMAPERAIRALH VSSTLGIPGQGNYAPHNAYLDALAARRRATGRSAVSVAWGPWDGGGMA - - - - - - AGDGVAERLRNHGVPGMDPELALAALE GAGVWGGGGQGAYGAANAFLDTLAEQRRARGLPATSISWGSWAGGGM - - - - - ADGAAGEHLRRRGIRPMPAASAILALQ $\ldots \ldots .490 \ldots \ldots 500 \ldots \ldots 510 \ldots \ldots 520 \ldots \ldots 530 \ldots \ldots 540 \ldots \ldots 50 \ldots \ldots 56$

RALDRDET - VLTVAPVAWERFFPLFSAARPRPLFEDLPQVR - - - ALSAPVPTTAGPAVEPGR - RGSGLGDL 583 RVMEDDET - VIGLMDIDWGTYHDVFTAGRPSHLFDRI PEVAR - - - LLADRAAPAATAVATSG - LAARLQGV 564 QMLENDDT - AAAITLMDWEMFAPAFTANRP SALLSTVPEAVS - - ALSDEGPADGGADAAVPP - LRARLDGL 574 QAIADTERPYVVIADIDWSKI - - - - - - EHTSQTSDLVS - - - - - AAREREPAVQRPTPPAE - - LHKTLAHQ 590 QVMAEPVA - TQVVADVDWQRFVADFTAVRPSRLLADLPEVR - - - -SLGEQRKDGPGGQGEEDG - LASKLAAL 531 TALADHDE - LCLVADVDWQRFREA - AGLRSTALLSSLPG - - - - - - AAGARTPAQTGPAEQDGQ - VRGQLAAL 561 NALDRAEV - CPIVIDVRWDRFLLAYTAQRPTRLFDEIDDARR - - - - - - AAPQAAAEP - - - RVGALASL 510 QALDNGDT - CVSIADVDWERFAVGFTAARPRPLLDELVT - . - . - PAVG - . . . . . . . . - . AVPAVQAA 467 SALGRDET - AITVADIDWDRFYLAYSSGRPOPLVEELPEVRRIIDARDSATSGOGGSSAOGANPLAERLAAA 512 EVLDQDET - CVSIADVDWDRFVPTFAATRATRLFDEVPAARK - - - - - - AMPA - NGPAEPGGS PFARNLAEL 565

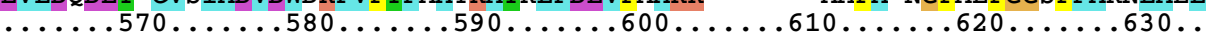




\section{Gene Synthesis}

The following genes were ordered from Integrated DNA Technologies as gBlocks.

Amp DE 2 (229 b.p):

CACACCAGGTCTCATGGAACCTAAGACCGCCGCTCCGGCGGGCACCGCAGACGCCTCAGACGCGGCGTTTTGGGAATTAGTTC AAAATCAGGATCTTGATGCGCTGGCCGGTGAACTGGGTATTCCCGCCGATGATGGCGCTGCCCGGCTGGGGGACGTACTTCCG GCGCTGTCAGCGTGGCGTACGCGTTCACTCACCCGTTCCCGTGCGGATGTGAGACCTGGTGTG

Amp KR $\mathrm{KR}_{2}(1492$ b.p):

CACACCAGGTCTCAGATGCTCTAAGATATCATATTGAATGGAACCGCGTCGCGGAGCCGGGAACTGCTCGTCCGGCGGGTCGT TTGCTGGCAGTGATTTCCCCCGACCATGCGGGCGCGCCTTGGGTCACGGCAGTCTTAGACGCCCTGGGACCGGACACGGTGCG TTTCGAAGCTAAGGGCACTGATCGTGCTGCATGGGCAGCCCAACTCGCCCAGCTGCGCGAGGATGAAGGGGAATTTCATGCAG TTGTGAGTCTGTTAGCGGCGGCGGAAGCCCTGCACACTGACCACGGCTCAGTCCCACTGGGCCTGGCCCAAACCTTACTGCTG GCCCAGGCGCTCGGCGATGCGGGCCTGACCGCGCCGTTATGGTGCCTCACCCGCGGGGGCGTTGCTGCAGGACGCGGTGATGT GCTCTCTTCACCGGTTCAGGGCGCTCTGTGGGGTCTGGGTCGCGTCATTGGCCTGGAACACCCCGACCGCTGGGGTGGCCTGA TTGATTTACCTGAAACAGTAGATACCCGGGCCGCGGCCCGCTTGACGGGGCTGCTTGCGGACGCGGGCGGTGAAGATCAACTG GCGATCCGCGGTTCGGGCGTACTCGCCCGTCGTCTGGCTCATGCAGCTCCTGCTGTGCCGGGGTCAGGCAAACGTCCGCCGGT GCATGGTAGTGTGCTTGTGACCGGAGGGACGGGCGGCATTGGTGGTCGCGTCGCTCGCCGCCTCGCGGAACAGGGAGCGGCAC ACCTGGTACTGACTTCACGTCGTGGTGCCGATGCCCCGGGCGCAGCCGAGCTGCGCGCTGAACTGGAACAACTCGGTGTACGC GTCACCATTGCCGCGTGTGACGCGGCGGATCGTGAAGCCCTCGCCGCACTGTTAGCGGAACTGCCGGAGGACGCTCCCCTGAC GGCTGTATTCCACAGCGCGGGTGTTGCCCACGATGATGCCCCGGTAGCCGACCTGACCCTGGGTCAGCTGGATGCGCTGATGC GTGCCAAGCTGACAGCAGCTCGTCATTTACATGAGCTCACCGCGGATCTTGACTTAGACGCCTTTGTACTTTTCTCATCAGGG GCGGCGGTATGGGGTTCCGGTGGTCAGCCGGGATATGCGGCCGCGAACGCGTATTTGGATGCACTGGCCGAGCACCGCCGCAG TCTGGGTTTGACCGCCAGCAGCGTAGCATGGGGTACCTGGGGCGAAGTAGGCATGGCTACCGACCCTGAAGTTCATGACCGTC TCGTTCGTCAGGGCGTTCTGGCGATGGAGCCAGAACATGCACTGGGGGCGCTTGACCAGATGCTTGAAAACGACGACACTGCC GCCGCGATTACGCTCATGGATTGGGAGATGTTTGCACCGGCCTTTACAGCGAATCGGCCAAGCGCATTACTGAGCACGGTTCC CGAGGCGGTTAGCGCATTAAGCGATGAGGGCCCAGCTGATGGAGGTGCAGATGCAGCTGTACCACCTTGAGACCTGGTGTG

Con $\mathrm{DE}_{2}(211$ b.p):

CACACCAGGTCTCATGGAAGCACCGCGGGCGTCGGATAGCGCGGCTCCGGCGGATGACGGGTTGTGGCGCGAAATTGAAGCGC AGGATAGCGCTGCTTTGGCCGACACCCTTGGTGTTGACCCTGGCGCCGTAGGTGAGGTTGTGCCGGCTTTGTCTGCTTGGCGG TCAGAACGTATCCGTGGCCGTCGCGCGGATATGAGACCTGGTGTG

Con $\mathrm{KR}_{2}$ (1471 b.p):

CACACCAGGTCTCAGATAGTTGGAGACATAGACTAGGATGGCGTCCGCTGACCCTGGCACCGCGCGCTAGTTTATCAGGGCGT TGGCTGGTTGTATTGCCGGCTACCGACTCTGGGGCCGATGTTCTGGCCGCGCTGACGGCAGCCGGTGCAGAACCAGTACCAGT AGTTGTGAGCGCACCGGATCAGGATCGTGAATCTCTTGCAACATCTCTTAGAGCTGCATCTGGTGCAGAACCTGTTGCAGGTG TATTCAGCTTGCTGGCTGAGGAGACGCACCCGCATCCTGCGCAGCCGGACGTACCGGCGGGCTTATCGCTTACCTTGGCGCTG GTGCAGGCCCTGGGGGATACGGGTACGGATGCACCTCTGTGGTGCGCTACCCGCGCACTGGCAGCAGTTGGTGGCGGAGAACG TCCTGGTGCTGGCGCACAGGCAGCTGTCGCTGGCCTCGGTCGCACTGTGGCACTTGAAAGACCGGACACGTGGGGAGGCTTGG TCGACTTGCCAGCGGTTCTGGATGAACGTGCTGCAGATCGTCTCGGTGCGGTATTGGCAACCGTTACCGATGAAGACCAGGTG GCTGTACGGTCTTCCGGCGTATACGGTCGTCGCCTTCTGCCTGTAGGTCCTGGAAGACCTGCAGAAGCTAGACATTCTCCATC AGGAACTGTTTTAATCACGGGTGGGACCGGCGGCACGGGTGCGCAGGTGGCCCGCGAGTTAGCGGAGCGCGGTGCTGCACATC TTCTACTGTTAAGTAGACGAGGTCCTGATGCTCCAGGAGCAGATGAACTGCGTGCTGAGTTAGCAGCTTTAGGTGCCCGCGTT ACCGTAGCAGCTTGTGATGTGGCAGATCGCGCACAGTTGGCGGCCGTCGTGGCTGCAATTCCAGAAGAAGAACCGCTGACAAC GGTGGTGCACGCAGCTGGTGTACTGGACGACGGGACTCTGGATGCGCTTGACCCTGCCCGTCTGGCGACGCTGATGCGTGTTA 
AAGTGACCGCGGCCCGGACTTTGCACGAGGTTACCGATGGTATTGGTCTGGCAGATTTCGTAGTATTCAGCAGCTTCATGGGC GTCTTGGGGAGCGCCGGACAGGGTAATTACGCCGCAGCAAATGCGGCGTTAGACGCTTTAGTCGCAGAGCGTCGCGCGGCAGG CCTGCCGGGCACTTCCGTGGCGTGGGGTGCGTGGGCCGGTGGCGGTATGGTTGACGATCGCATCGCGGAGCGCTTACGGCGCT TGGGCATTACGCCTATGGAGCCGCGTGCGGCGGTGCGTGCAATGACTACCGCGCTGGCCGATCATGATGAACTCTGTTTAGTT GCCGACGTGGATTGGCAACGCTTTCGTGAAGCTGCGGGCCTTCGCAGTACTGCGTTGCTGTCATCGTTGCCAGGTGCTGCAGG AGCTAGAACTCCTGCTCAAACAGGTCCAGCTGAACAAGATGGTCAGTGAGACCTGGTGTG

The following genes were ordered from Genewiz and delivered in pUC57-Kan flanked by a 5'-GCTAGCT sequence containing a BmtI cut site and a 3'-GGTACCGC sequence containing a Acc65I cut site.

Amp KR $\mathrm{KR}_{1}$ (1449 b.p.):

GATAGCTGGCGTTATCGTGTGGTGTGGGAACCTCTGGCCCAGATTCCGCGCGCAACCCTGGATGGCACCTGGTTACTGGTTAG CGCAGGCGGTGTGGATGACGATGTTGCCGAAGTTCTGGAAGCAGGTGGCGCCGAAGTGCGTCGCCTGGTTCTGGATGAAACAT GCACCGATCCGGCCGTGCTGCGTGAACACCTGACCGATACAGATGGCTTAACCGGCATCGTTAGCGTGCTGGCAGATGCAGAA GAAGGTTGCGTTCGCCATCCTGGTCTGACCCTGGGCCTGGCACTGAGTGTGAGCCTGGCACAGGCCTTAGGTGAGGCAGATGT GACCGCCCCGCTGTGGTTTCTGACCTGTGGTGCCTTCAGCACCGGCCCGAGCGATACAGTGACACGCCCGTTACAGAGCCAGA TTGCAGGCCTGGGCTGGACCGTGGCCGTTGAACATCCGCATCGTTGGGGTGGTGGTGTTGACCTGCCTGAAGCATTAGATGCC CGCGCCGCACAGCGCTTAGTTGCAGCACTGGCCGGTGCCCTGGGCGATGATGATCAGCTGGCAGTTCGTCCCGCTGGTGTTCT GGCCCGTCGTATTGTTCGCGCAAGTGGCGATACCCGCCGCAAAGCACGTAGCTGGAAACCGCGCGGCACTACACTGGTTACCG GCGGCAGTGGTACACTGGCACCGGGTCTGGCCCGCCATTTAGCCGCACAGGGTGCAGAACATCTGGTGTTACTGAGCCGTCGT GGTGCCGATGCCCCTGGCGCAGCAGAACTGGCAGCAGAGTTACAAGCAGCCGGCACTGAAGTTCGTTTTGCCGCCTGCGATAT TACCGACCCGGATGCCGTGGCAGCCTTACTGGCCGATCTGAAGGCCGAAGGCCGTACCGTTCGTACCGTGGTGCATACCGCCG CCGTTATCGAACTGGCCGCACTGGCCGATACAACCGTGGACGCATTCGCCGATGTGGTTCATGCCAAGGTTACCGGTGCACGC ATTCTGGACGAGCTGCTGGACGATGAAGAACTGGATGATTTTGTTCTGTATAGTAGCACCGCAGGTATGTGGGGCAGTGGCGT GCATGCAGCCTACGTTGCCGGCAACGCCTATCTGAGCGCACTGGCAGAACAACGCCGCGCACGTGGTGCACGCGCCACCAGCA TTCACTGGGGTAAATGGCCGGATGACCTGGAACGTGAACTGGCAGATCCTCACCAGATCCGTCGTAGCGGCCTGGAATATTTA GATCCTGAATTAGCTATGACCGCCCTGACACGTGTGATGGAGGATGATGAAACTGTGATCGGTCTGATGGACATTGACTGGGG CACATACCATGACGTTTTTACCGCAGGCCGTCCGAGCCATCTGTTCGATCGCATTCCGGAGGTGGCACGTCTGTTAGCCGACC GTGCCGCACCGGCAGCAACCGCAGTTGCAACCAGTGGT

Pik KR $\mathrm{KR}_{1}$ (1443 b.p.):

GATGACTGGCGCTATCGCATTGACTGGAAACGTCTGCCTGCCGCCGAAGGCAGTGAACGTACCGGTCTGAGCGGTCGCTGGTT AGCCGTTACCCCTGAAGATCACAGTGCCCAGGCAGCCGCCGTGTTAACAGCCCTGGTGGATGCCGGTGCAAAAGTTGAGGTGC TGACCGCAGGCGCCGATGATGATCGCGAAGCATTAGCCGCCCGTCTGACCGCACTGACAACCGGTGACGGTTTTACCGGCGTT GTGAGCCTGCTGGATGGCCTGGTGCCGCAGGTTGCCTGGGTTCAGGCACTGGGCGATGCAGGTATTAAGGCACCGCTGTGGAG CGTTACCCAGGGTGCCGTGAGTGTTGGTCGTCTGGATACCCCGGCAGACCCGGATCGTGCAATGTTATGGGGCCTGGGCCGCG TTGTTGCACTGGAGCATCCGGAACGTTGGGCAGGTCTGGTTGATCTGCCGGCCCAGCCTGATGCAGCAGCCCTGGCACATCTG GTGACCGCTTTATCTGGCGCAACCGGCGAAGATCAGATTGCAATCCGCACCACCGGTCTGCATGCCCGTCGTCTGGCACGTGC ACCGTTACACGGTCGCCGCCCGACACGTGATTGGCAGCCTCATGGCACTGTTCTGATCACCGGCGGTACAGGCGCCTTAGGTA GCCATGCCGCACGTTGGATGGCCCATCATGGTGCCGAACATCTGCTGCTGGTGAGCCGTAGTGGCGAGCAAGCACCTGGCGCC ACACAGCTGACAGCCGAACTGACAGCCAGTGGTGCCCGCGTTACCATCGCAGCCTGCGATGTGGCAGATCCGCATGCAATGCG CACCCTGCTGGACGCCATTCCGGCAGAAACCCCGCTGACAGCCGTGGTGCATACCGCAGGTGCCCTGGATGATGGCATTGTGG ACACCCTGACCGCAGAACAGGTGCGCCGTGCCCATCGTGCAAAAGCCGTGGGTGCCAGTGTGTTAGATGAGCTGACCCGCGAT CTGGACCTGGATGCCTTCGTGCTGTTTAGCAGCGTGAGTAGTACCCTGGGTATTCCGGGTCAGGGTAATTACGCCCCGCACAA CGCCTATCTGGACGCACTGGCAGCACGTCGTCGCGCAACCGGTCGTAGCGCAGTGAGTGTGGCATGGGGTCCGTGGGATGGTG 
GTGGTATGGCAGCCGGCGATGGTGTTGCCGAACGCCTGCGTAACCATGGCGTTCCGGGTATGGACCCTGAACTGGCCCTGGCC GCACTGGAAAGCGCCTTAGGCCGCGACGAAACCGCCATCACCGTGGCCGATATCGATTGGGACCGTTTCTACCTGGCATACAG CAGTGGCCGCCCTCAACCGCTGGTTGAAGAGCTGCCGGAAGTTCGCCGCATTATTGATGCACGCGACAGTGCCACCAGCGGTC AAGGCGGTAGTAGCGCACAGGGCGCCAATCCG

Spn $\mathrm{KR}_{3}$ (1440 b.p.):

GACGGTTGGCGCTATCGTGTTGATTGGGTGCGTGTTGCCCCTCAGCCGGTTCGTCGCACACGTGAAACCTGGCTGCTGGTTGT TCCGCCGGGTGGTATTGAAGAAGCCCTGGTGGAACGCCTGACCGATGCCCTGAATACCCGTGGCATTAGCACCCTGCGCCTGG ATGTTCCGCCGGCAGCAACAAGTGGCGAATTAGCCACCGAACTGCGTGCCGCCGCAGATGGTGATCCGGTTAAGGCCATTCTG AGCCTGACCGCACTGGATGAACGTCCGCATCCGGAATGTAAGGATGTGCCTAGCGGCATTGCCCTGTTACTGAATCTGGTGAA GGCCCTGGGTGAGGCCGATCTGCGCATCCCGCTGTGGACAATTACTCGTGGTGCAGTGAAAGCCGGTCCTGCCGATCGTCTGC TGCGTCCTATGCAGGCCCAGGCATGGGGTTTAGGCCGTGTTGCAGCCCTGGAACATCCGGAACGTTGGGGTGGCCTGATTGAT CTGCCGGACAGCCTGGATGGCGACGTGCTGACCCGTCTGGGTGAAGCCCTGACCAATGGTCTGGCAGAAGATCAGCTGGCAAT CCGTCAAAGCGGTGTGCTGGCACGCCGTTTAGTTCCTGCCCCGGCAAATCAGCCGGCAGGCCGTAAATGGCGCCCTCGCGGTA GCGCACTGATTACCGGCGGTCTGGGTGCAGTGGGTGCACAGGTTGCACGTTGGCTGGCCGAAATCGGCGCAGAACGCATTGTG CTGACCAGCCGTCGCGGCAATCAAGCAGCCGGTGCAGCCGAACTGGAAGCCGAGCTGCGTGCACTGGGCGCACAGGTTAGCAT TGTGGCCTGCGATGTGACCGATCGTGCCGAAATGAGTGCCCTGCTGGCAGAGTTTGATGTGACAGCCGTGTTCCATGCAGCAG GCGTGGGTCGCCTGCTGCCGTTAGCAGAAACCGACCAGAATGGCCTGGCAGAAATTTGTGCCGCCAAAGTGCGCGGCGCACAA GTTCTGGACGAACTGTGCGACAGCACCGATCTGGACGCCTTTGTGCTGTTTAGTAGCGGTGCAGGCGTTTGGGGTGGTGGCGG TCAGGGCGCTTATGGTGCAGCAAATGCATTCCTGGATACCCTGGCAGAGCAGCGTCGTGCACGCGGTTTACCGGCAACCAGCA TTAGCTGGGGTAGCTGGGCAGGTGGTGGTATGGCAGATGGCGCCGCAGGTGAACATCTGCGTCGTCGCGGTATCCGCCCTATG CCGGCAGCCAGTGCCATTCTGGCCTTACAAGAAGTGCTGGACCAGGATGAAACTTGTGTTAGCATTGCCGACGTTGATTGGGA CCGCTTTGTTCCGACATTTGCAGCAACCCGTGCAACCCGTCTGTTCGATGAGGTGCCGGCAGCACGCAAAGCAATGCCTGCAA ATGGTCCGGCAGAACCGGGTGGTAGCCCG

The following genes were ordered from Genscript.

Ave $\mathrm{KR}_{1}$ (1464 b.p.):

AATACCTGGACGTACCAGGAAACGTGGAAACCGCTGACGCTGCCGACCACCCATCAACCGCACCAGACCTGGTTGATCGCCAT CCCGGAAACGCAAACCCACCATCCACATATTACCAACATCCTGACGAACTTGCACCATCACGGCATCACCCCAATCCCTCTGA CCCTGAACCATACGCACACCAATCCTCAGCATCTGCATCATACCCTGCACCACACCCGTCAGCAGGCACAAAACCATACCACG GGTGCAATTACCGGTCTGCTGAGCCTGTTGGCACTGGATGAAACGCCGCACCCGCACCACCCGCATACCCCTACGGGCACGCT GCTGAATTTGACGCTGACCCAGACCCACACGCAAACGCATCCGCCAACTCCGCTGTGGTATGCTACGACGAACGCAACGACGA CGCATCCTAACGATCCATTGACCCACCCGACCCAGGCGCAGACCTGGGGCCTGGCGCGTACGACCTTGCTGGAGCACCCTACC CATACCGCGGGTATCATTGATCTGCCGACCACGCCGACGCCTCATACCTTGCAGCATTTGACGCAGACGCTGACCCAGCCTCA CCATCAGACGCAGCTGGCGATCCGTACTACCGGCACTCACACGCGCCGTCTGACCCCGACGACTCTGACGCCGACCCACCAGC CGCCGACCCCGACCCCGCATGGTACGACGCTGATCACGGGTGGCACGGGTGCGCTGGCGACGCACCTGACGCATCACCTGACG ACCCACCAACCTACGCAGCACCTGCTGCTGACCAGCCGTACTGGTCCTCACACCCCGCACGCGCAGCATCTGACTACCCAGCT GCAACAAAAGGGCATCCACCTGACCATTACCACGTGTGATACCTCTAATCCGGACCAGTTGCAACAGCTGCTGAACACGATTC CGCCGCAGCACCCGCTGACTACCGTGATTCACACTGCTGGTATTCTGGACGACGCGACCCTGACCAACCTGACCCCAACCCAG CTGAATAACGTCCTGCGTGCGAAAGCGCACTCTGCCCATCTGCTGCACCAGCTGACCCAACACACGCCGCTGACTGCGTTTGT GCTGTACAGCTCTGCCGCTGCGACCTTCGGTGCTCCGGGCCAGGCCAATTACGCCGCAGCGAACGCGTATCTGGATGCTCTGG CCCACCACCGTCACACGCATCACTTGCCGGCGACCTCTATTGCTTGGGGCACGTGGCAGGGTAATGGCCTGGCGGACAGCGAT AAAGCTCGTGCGTACCTGGACCGTCGCGGTTTCCGCCCGATGTCCCCAGAACTGGCAACCGCTGCAGTTACTCAGGCGATCGC GGACACGGAGCGTCCGTACGTTGTTATTGCAGATATCGATTGGAGCAAGATTGAGCACACGAGCCAGACGAGCGATCTGGTTA GCGCAGCCCGTGAACGTGAGCCGGCAGTGCAACGTCCTACCCCGCCTGCCGAG 
Ery $\mathrm{KR}_{1}$ (1422 b.p.):

TCTGCACTGCGTTATCGCATTGAATGGCGTCCGACCGGTGCTGGTGAACCGGCACGTCTGGATGGTACGTGGCTGGTTGCGAA ATACGCAGGCACCGCTGACGAAACGTCAACCGCAGCTCGCGAAGCGCTGGAATCGGCAGGTGCTCGTGTTCGCGAACTGGTTG TCGATGCACGTTGCGGTCGTGACGAACTGGCAGAACGTCTGCGCAGCGTCGGTGAAGTGGCGGGCGTTCTGTCTCTGCTGGCC GTGGATGAAGCAGAACCGGAAGAAGCGCCGCTGGCGCTGGCCAGTCTGGCCGATACCCTGTCCCTGGTGCAGGCTATGGTTAG CGCGGAACTGGGTTGTCCGCTGTGGACGGTGACCGAATCTGCAGTTGCTACCGGTCCGTTTGAACGTGTTCGTAACGCAGCAC ATGGCGCGCTGTGGGGTGTCGGCCGCGTGATTGCCCTGGAAAATCCGGCAGTCTGGGGCGGTCTGGTCGATGTGCCGGCAGGT AGTGTTGCAGAACTGGCCCGTCACCTGGCAGCTGTGGTTTCCGGCGGTGCAGGTGAAGATCAGCTGGCACTGCGTGCTGACGG TGTGTACGGTCGTCGCTGGGTTCGTGCAGCAGCACCGGCAACCGATGACGAATGGAAACCGACGGGCACCGTTCTGGTCACCG GCGGCACCGGCGGCGTGGGCGGCCAAATCGCACGTTGGCTGGCACGTCGCGGTGCTCCGCATCTGCTGCTGGTTAGCCGTTCT GGTCCGGATGCAGACGGTGCAGGCGAACTGGTTGCGGAACTGGAAGCACTGGGTGCTCGCACCACGGTCGCTGCATGCGATGT GACCGACCGTGAATCAGTGCGTGAACTGCTGGGCGGTATTGGCGATGACGTTCCGCTGTCGGCCGTCTTTCATGCCGCAGCTA CGCTGGATGACGGCACCGTGGATACGCTGACCGGCGAACGTATCGAACGCGCGTCACGTGCCAAAGTTCTGGGTGCGCGCAAC CTGCACGAACTGACGCGTGAACTGGATCTGACCGCATTTGTGCTGTTCTCATCGTTTGCGAGTGCATTCGGTGCGCCGGGTCT GGGCGGTTATGCGCCGGGTAATGCCTACCTGGATGGCCTGGCGCAGCAACGTCGCAGTGACGGTCTGCCGGCAACGGCTGTTG CATGGGGCACCTGGGCAGGTTCCGGTATGGCAGAAGGTCCGGTTGCAGATCGTTTTCGTCGCCATGGCGTCATTGAAATGCCG CCGGAAACCGCATGCCGCGCTCTGCAGAACGCACTGGATCGTGCTGAAGTGTGTCCGATTGTTATCGATGTCCGCTGGGACCG TTTCCTGCTGGCGTATACGGCACAGCGTCCGACCCGTCTGTTTGATGAAATCGATGACGCACGTCGCGCAGCACCGCAAGCAG CTGCGGAACCG

Ery $\mathrm{KR}_{6}$ (1311 b.p.):

GACTCCCGCTATCGTGTTGATTGGCGCCCGCTGGCCACCACGCCGGTGGATCTGGAAGGCGGTTTTCTGGTGCATGGCTCAGC ACCGGAAAGCCTGACCTCTGCGGTTGAAAAAGCCGGCGGTCGTGTGGTTCCGGTTGCTTCGGCGGATCGTGAAGCACTGGCAG CTGCGCTGCGTGAAGTTCCGGGCGAAGTCGCGGGTGTGCTGAGTGTTCACACCGGTGCGGCAACCCACCTGGCACTGCACCAG TCCCTGGGTGAAGCAGGTGTTCGTGCTCCGCTGTGGCTGGTCACGAGCCGTGCGGTGGCACTGGGCGAATCTGAACCGGTTGA TCCGGAACAGGCCATGGTCTGGGGCCTGGGTCGTGTGATGGGTCTGGAAACCCCGGAACGTTGGGGCGGTCTGGTGGATCTGC CGGCTGAACCGGCCCCGGGTGATGGTGAAGCATTTGTCGCATGCCTGGGCGCGGATGGTCATGAAGACCAGGTGGCAATCCGC GATCACGCTCGTTATGGCCGTCGCCTGGTTCGTGCACCGCTGGGTACGCGTGAAAGCAGCTGGGAACCGGCTGGCACCGCCCT GGTGACCGGCGGTACGGGTGCCCTGGGCGGTCATGTGGCGCGCCACCTGGCCCGTTGCGGCGTTGAAGATCTGGTGCTGGTTT CACGTCGCGGCGTGGACGCCCCGGGTGCTGCGGAACTGGAAGCCGAACTGGTTGCCCTGGGTGCAAAAACCACGATTACCGCA TGTGATGTGGCTGACCGCGAACAGCTGTCTAAACTGCTGGAAGAACTGCGCGGTCAGGGTCGCCCGGTTCGCACCGTTGTGCA TACGGCGGGCGTGCCGGAAAGCCGCCCGCTGCACGAAATCGGTGAACTGGAAAGCGTGTGCGCCGCAAAAGTCACGGGCGCAC GTCTGCTGGATGAACTGTGTCCGGACGCTGAAACCTTTGTTCTGTTCAGTTCCGGTGCGGGTGTCTGGGGCTCAGCCAACCTG GGTGCCTATTCGGCTGCGAATGCCTACCTGGATGCTCTGGCGCATCGTCGCCGTGCCGAAGGTCGTGCCGCAACCAGTGTGGC ATGGGGTGCGTGGGCAGGTGAGGGTATGGCCACCGGTGATCTGGAAGGTCTGACCCGCCGTGGCCTGCGTCCGATGGCCCCGG AACGCGCTATTCGTGCGCTGCACCAGGCCCTGGATAACGGCGACACCTGTGTGAGCATCGCAGATGTTGACTGGGAACGCTTT GCTGTTGGTTTTACGGCTGCGCGCCCGCGTCCGCTGCTGGATGAACTGGTGACCCCGGCCGTCGGT 


\section{Primers}

Table 2: Primers used to probe the importance of the DE in KR domain exchange experiments.

\begin{tabular}{|c|c|c|}
\hline Primer & Sequence & Target \\
\hline debb_1f & CACACCAGGTCTCAACCTCGTGGTAGCGGCCTGGGC & pSY044 \\
\hline nodebb_2f & CACACCAGGTCTCATCAGCGTGGTAGCGGCCTGGGC & pSY044 \\
\hline backbone_r & CACACCAGGTCTCATCCAGCCAGTAACGCTGATGCTGAAATGCG & pSY044 \\
\hline lipde1_f & CACACCAGGTCTCATGGATAGCACCAGCAAACACTGG & pSY044 \\
\hline lipde1_r & CACACCAGGTCTCACATCAACAGCCGCTGCCGTACGC & pSY044 \\
\hline ampde $2 \_f$ & CACACCAGGTCTCATGGAACC & Amp $\mathrm{DE}_{2}$ \\
\hline ampde2_r & CACACCAGGTCTCACATCCGC & Amp $\mathrm{DE}_{2}$ \\
\hline ampkr2_f & CACACCAGGTCTCAGATGC & Amp $\mathrm{KR}_{2}$ \\
\hline ampkr2_r & CACACCAGGTCTCAAGGTGG & Amp $\mathrm{KR}_{2}$ \\
\hline conde $2 \_f$ & CACACCAGGTCTCATGGAAGCACCG & Con $\mathrm{DE}_{2}$ \\
\hline conde2_r & CACACCAGGTCTCATATCCGCGCG & Con $\mathrm{DE}_{2}$ \\
\hline conkr2_f & CACACCAGGTCTCAGATAGTTGGAGACA & Con $\mathrm{KR}_{2}$ \\
\hline conkr2_r & CACACCAGGTCTCACTGACCATCTTGT & Con $\mathrm{KR}_{2}$ \\
\hline dedel_f & GACGCATGGCGTTACCGCAC & pSY044 \\
\hline dedel_r & GTCACCAGTGTTTGCAGTGGTGCTATC & pSY044 \\
\hline
\end{tabular}


Table 3: Primers used to investigate the generalizability of the KR domain exchange strategy.

\begin{tabular}{|c|c|c|}
\hline Primer & Sequence & Target \\
\hline debb_f & CACACCAGGTCTCACGTGGTAGCGGCCTGGGC & pSY044 \\
\hline debb_r & CACACCAGGTCTCAAACAGCCGCTGCCGTACGC & pSY044 \\
\hline avekr1_f & CACACCAGGTCTCAGGCTAATACCTGGACGTACCAGGAAACGTGGA & Ave $\mathrm{KR}_{1}$ \\
\hline avekr1_r & CACACCAGGTCTCACTGCCTCGGCAGGCGGGGTAGGAC & Ave $\mathrm{KR}_{1}$ \\
\hline erykr1_f & CACACCAGGTCTCATGTTTCTGCACTGCGTTATCGCATTGAATGG & Ery $\mathrm{KR}_{1}$ \\
\hline erykr1_r & CACACCAGGTCTCACACGCGGTTCCGCAGCTGCTTGC & Ery $\mathrm{KR}_{1}$ \\
\hline borkr1_f & CACACCAGGTCTCATGTTGGCGGTTGGCGTTATCGTATTCACTGG & pARH100 \\
\hline borkr1_r & CACACCAGGTCTCACACGACCGTCCTCTTCACCTTGGCCG & pARH100 \\
\hline spnkr3_f & CACACCAGGTCTCATGTTGACGGTTGGCGCTATCGTGTTGATTGG & Spn $\mathrm{KR}_{3}$ \\
\hline spnkr3_r & CACACCAGGTCTCACACGCGGGCTACCACCCGGTTCTGC & Spn $\mathrm{KR}_{3}$ \\
\hline $\operatorname{ampkr1} \mathrm{f}$ & CACACCAGGTCTCATGTTGATAGCTGGCGTTATCGTGTGGTGTGG & Amp $\mathrm{KR}_{1}$ \\
\hline ampkr1_r & CACACCAGGTCTCACACGACCACTGGTTGCAACTGCGG & Amp $\mathrm{KR}_{1}$ \\
\hline pikkr1_f & CACACCAGGTCTCATGTTGATGACTGGCGCTATCGCATTGACTGG & $\mathrm{Pik} \mathrm{KR}_{1}$ \\
\hline pikkr1_r & CACACCAGGTCTCACACGCGGATTGGCGCCCTGTGCG & $\mathrm{Pik} \mathrm{KR}_{1}$ \\
\hline erykr6_f & CACACCAGGTCTCATGTTGACTCCCGCTATCGTGTTGATTGGCG & Ery $\mathrm{KR}_{6}$ \\
\hline erykr6_r & CACACCAGGTCTCACACGACCGACGGCCGGGGTCACC & Ery $\mathrm{KR}_{6}$ \\
\hline
\end{tabular}




\section{Plasmids}

Table 4: Expression plasmids used in this study.

\begin{tabular}{|c|c|c|c|}
\hline Plasmid & Description & Parent & Antibiotic resistance \\
\hline pSY044 & Lip $\mathrm{KR}_{1}$ & $\mathrm{pET} 30 \mathrm{~b}$ & Kanamycin \\
\hline pCE001 & Amp $\mathrm{KR}_{2}$ & pSY044 & Kanamycin \\
\hline $\mathrm{pCE} 002^{a}$ & Amp DE, $\mathrm{KR}_{2}$ & pSY044 & Kanamycin \\
\hline pCE003 & Con $\mathrm{KR}_{2}$ & pSY044 & Kanamycin \\
\hline $\mathrm{pCE} 004^{a}$ & Con $\mathrm{DE}, \mathrm{KR}_{2}$ & pSY044 & Kanamycin \\
\hline pCE006 & Ave $\mathrm{KR}_{1}$ & pSY044 & Kanamycin \\
\hline pCE007 & Ery $\mathrm{KR}_{1}$ & pSY044 & Kanamycin \\
\hline pCE008 & Bor $\mathrm{KR}_{1}$ & pSY044 & Kanamycin \\
\hline pCE009 & Spn $\mathrm{KR}_{3}$ & pSY044 & Kanamycin \\
\hline pCE010 & Amp $\mathrm{KR}_{1}$ & pSY044 & Kanamycin \\
\hline pCE012 & $\mathrm{Pik} \mathrm{KR}_{1}$ & pSY044 & Kanamycin \\
\hline pCE015 & Ery $\mathrm{KR}_{6}$ & pSY044 & Kanamycin \\
\hline pCE022 & No DE (RPA to AAV), Lip $\mathrm{KR}_{1}$ & pSY044 & Kanamycin \\
\hline
\end{tabular}




\section{Soluble Expression of (-DE)LipPks1+TE (SDS-PAGE)}

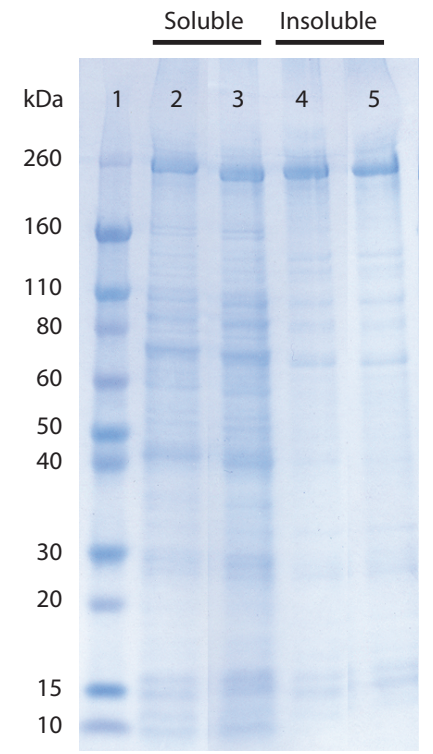

Figure 2: SDS-PAGE analysis of recombinant LipPks1+TE (259 kDa) and (-DE)LipPks1+TE $(253 \mathrm{kDa})$. Proteins were resolved using a 4-15\% Tris-Glycine gel and stained with Coomassie blue. Lane 1, molecular weight marker; Lanes 2 and 4, LipPks1+TE; Lanes 3 and 5, (-DE)LipPks1+TE. 


\section{Purity of LipPks1+TE Variants (SDS-PAGE)}

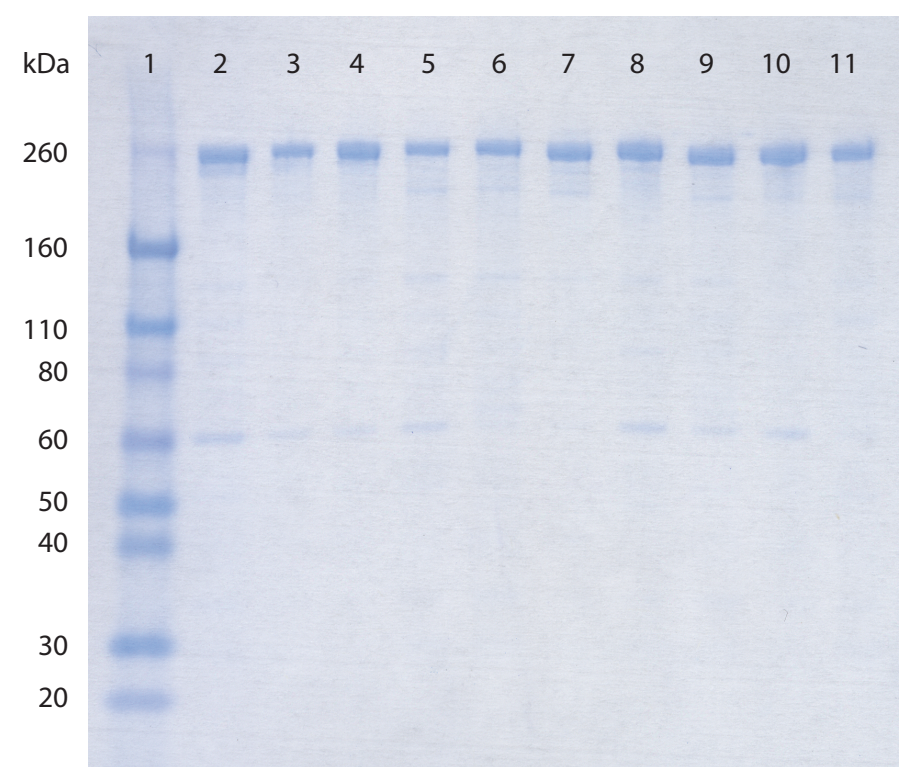

Figure 3: SDS-PAGE analysis of recombinant LipPks1+TE (259 kDa) and variants purified to $>90 \%$ purity. Proteins were resolved using a 4-15\% Tris-Glycine gel and stained with Coomassie blue. Lane 1, molecular weight marker; Lanes 211 (in order), LipPks1+TE, (Amp KR $)$ LipPks1+TE, (Amp DE,KR 2 LipPks1+TE, (Con $\left.\mathrm{KR}_{2}\right)$ LipPks1+TE, $(\text { Con DE,KR })_{2}$ LipPks1+TE, $($ Ery KR 1$) L i p P k s 1+T E$, (Bor KR 1$)$ LipPks1+TE, $($ Spn KR 3$)$ LipPks1+TE, (Amp KR $)$ LipPks1+TE, $($ Ery KR 6 )LipPks1+TE 


\section{Production of 3a-d by LipPks1+TE Variants (LC-MS Chro- matograms)}

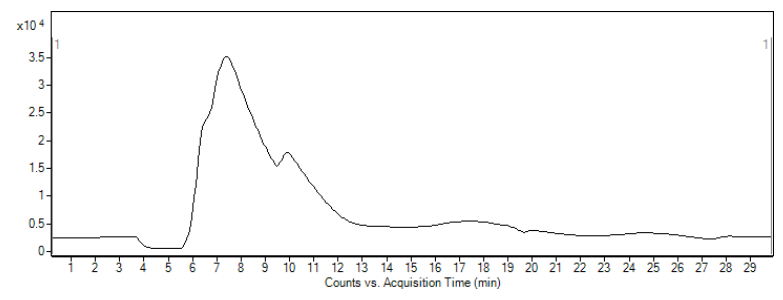

(a) $0 \mu \mathrm{M} \mathrm{3a}$.

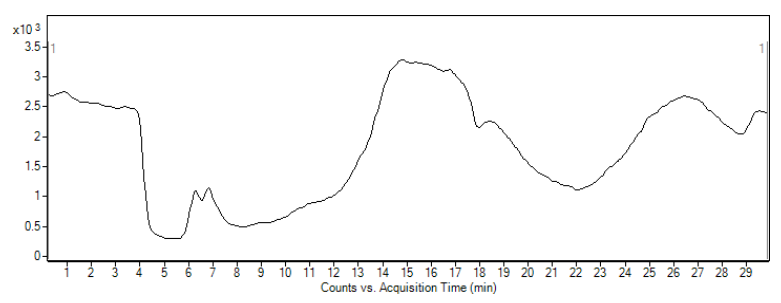

(c) $0 \mu \mathrm{M} \mathbf{3 b}$.

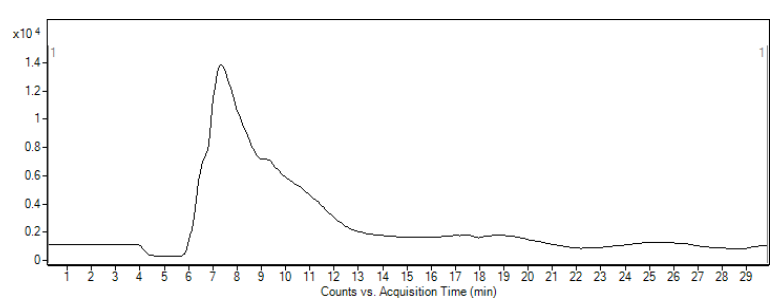

(e) $0 \mu \mathrm{M} 3 \mathbf{c}$.

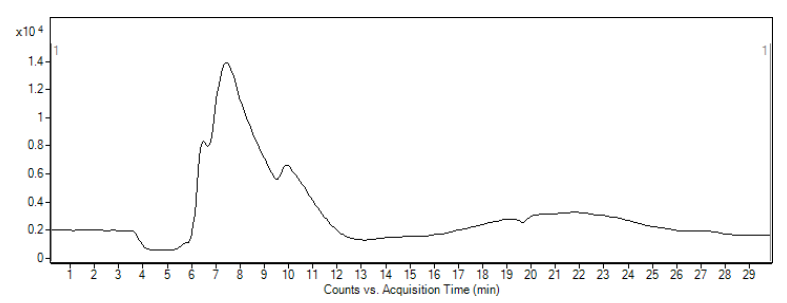

(g) $0 \mu \mathrm{M} \mathbf{3 d}$.

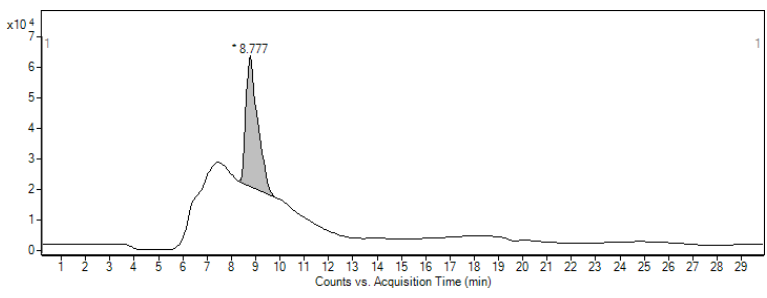

(b) $25 \mu \mathrm{M} \mathrm{3a}$.

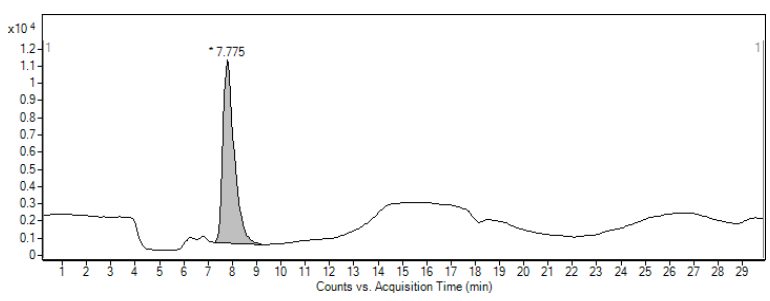

(d) $25 \mu \mathrm{M} 3 \mathbf{b}$.

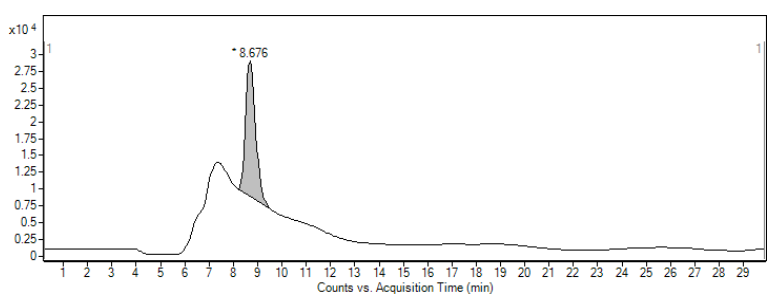

(f) $25 \mu \mathrm{M} \mathrm{3c}$.

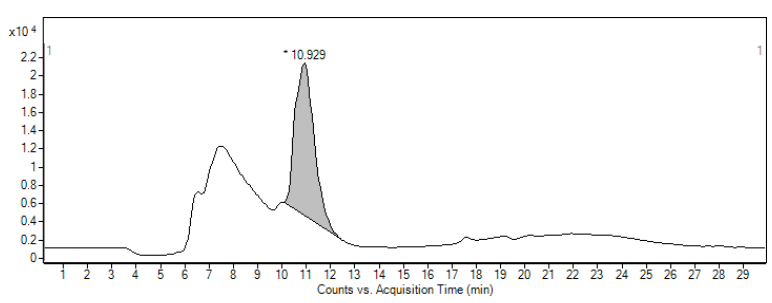

(h) Qualitative 3d.

Figure 4: Authentic hydroxyacid standards 3a-d. 


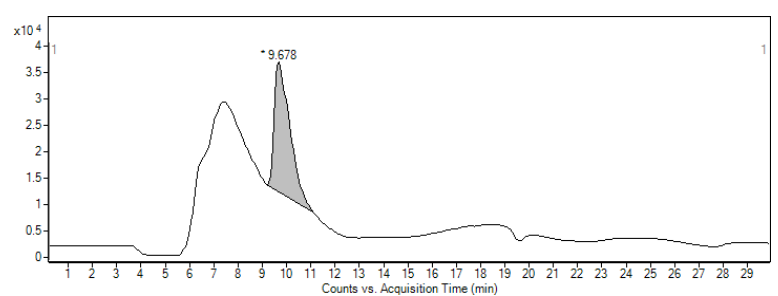

(a) Wild-type LipPks1+TE.

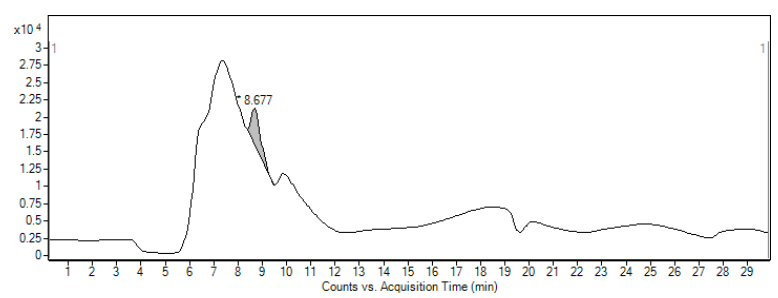

(c) $\left(\mathrm{Amp} \mathrm{DE}, \mathrm{KR}_{2}\right) \mathrm{LipPks} 1+\mathrm{TE}$.

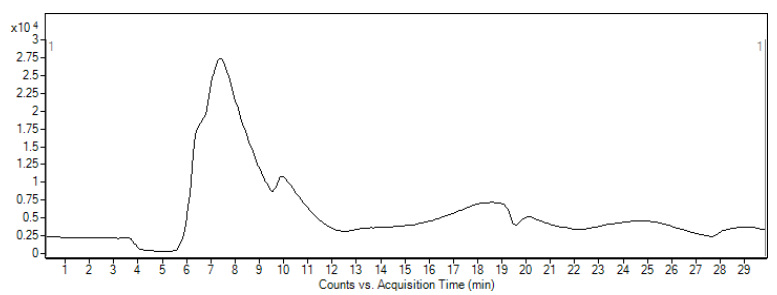

(e) (Con DE,KR 2 LipPks1+TE.

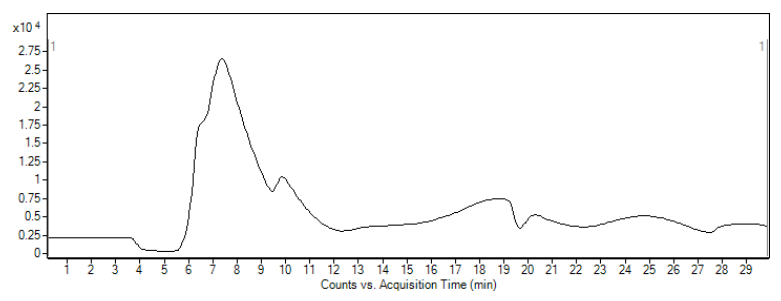

(g) $\left(\right.$ Bor $\left.\mathrm{KR}_{1}\right) \mathrm{LipPks} 1+\mathrm{TE}$.

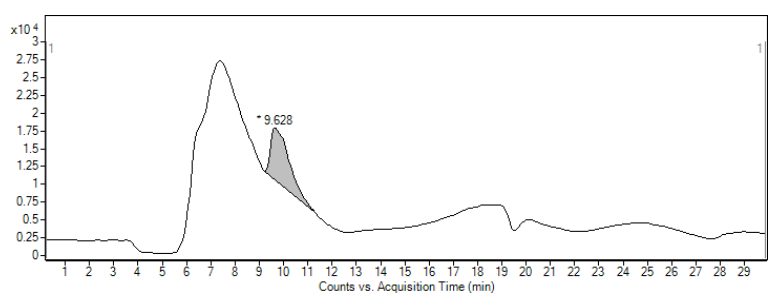

(i) $\left(\mathrm{Amp} \mathrm{KR}_{1}\right) \mathrm{LipPks1+TE}$.

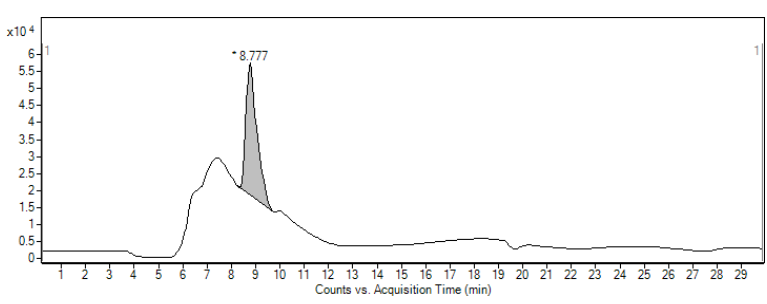

(b) $\left(\right.$ Amp KR $\left.\mathrm{KR}_{2}\right) \mathrm{LipPks1+TE}$.

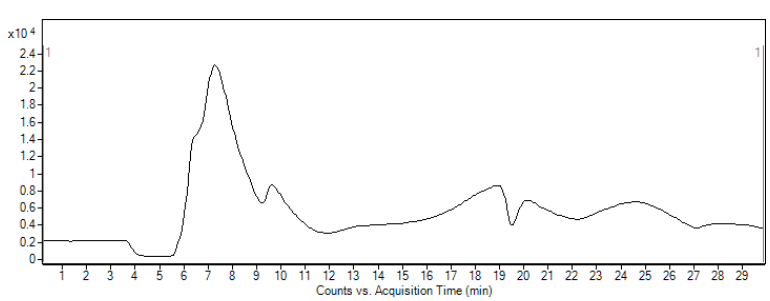

(d) $\left(\right.$ Con $\left.\mathrm{KR}_{2}\right) \mathrm{LipPks} 1+\mathrm{TE}$.

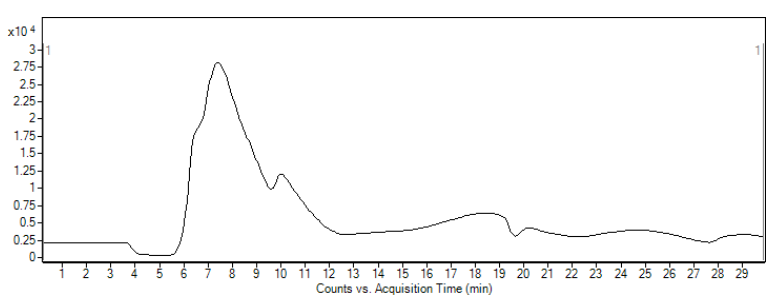

(f) $\left(\right.$ Ery $\left.\mathrm{KR}_{1}\right) \mathrm{LipPks} 1+\mathrm{TE}$.

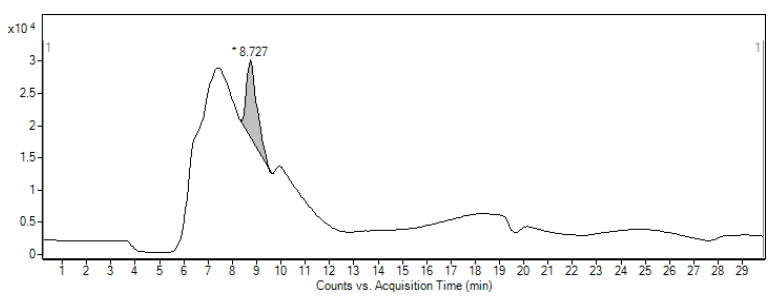

(h) $\left(\mathrm{Spn} \mathrm{KR}_{3}\right) \mathrm{LipPks1+TE}$.

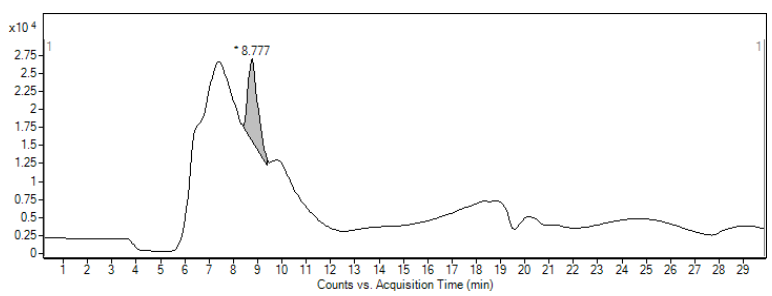

(j) (Ery $\left.\mathrm{KR}_{6}\right) \mathrm{LipPks1+TE}$.

Figure 5: Overnight production of $\mathbf{3 a}$. 


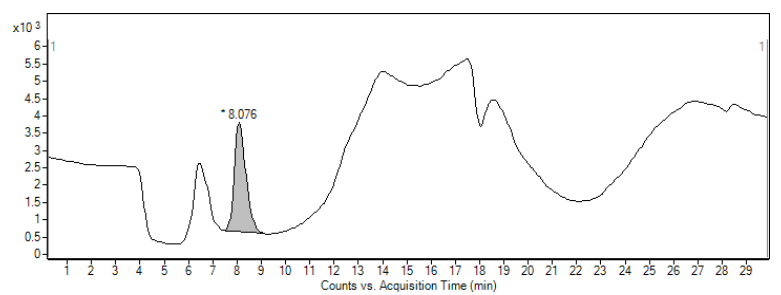

(a) Wild-type LipPks1+TE.

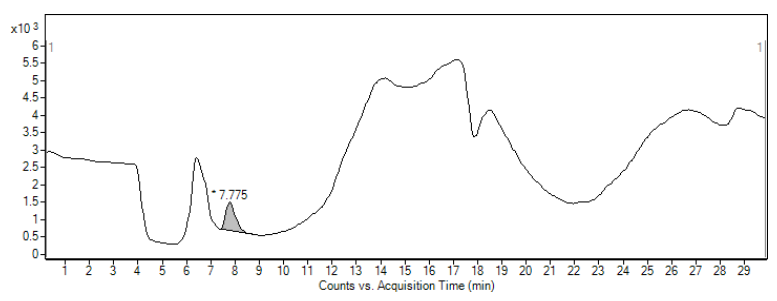

(c) (Amp DE,KR 2$) \mathrm{LipPks} 1+\mathrm{TE}$.

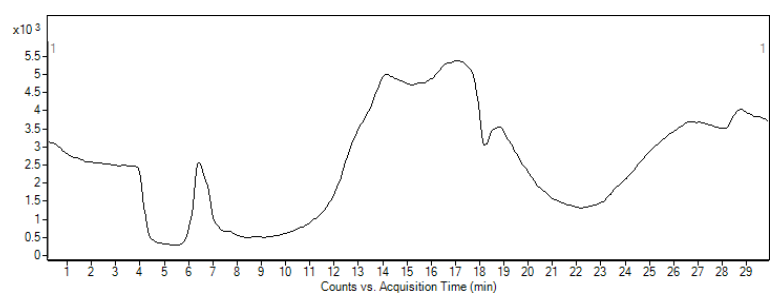

(e) (Con DE,KR 2 LipPks1+TE.

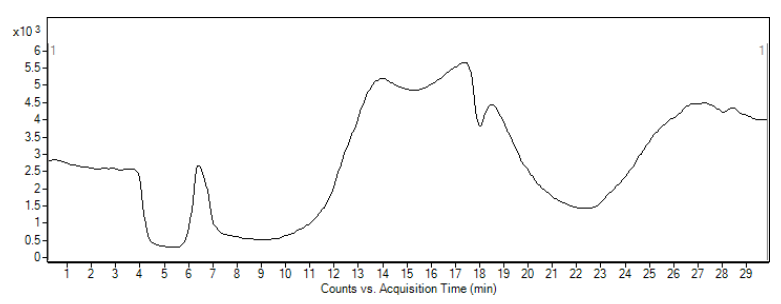

(g) $\left(\right.$ Bor $\left.\mathrm{KR}_{1}\right) \mathrm{LipPks1+TE}$.

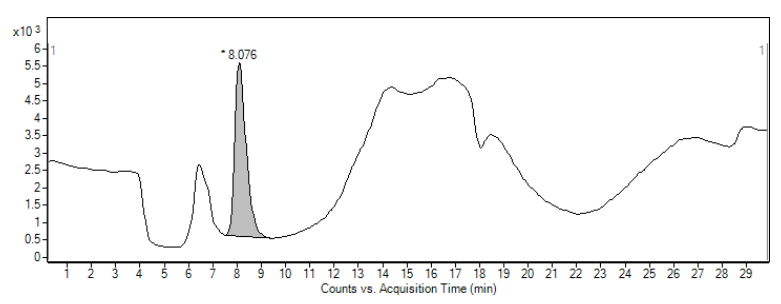

(i) $\left(\operatorname{Amp} \mathrm{KR}_{1}\right) \mathrm{LipPks1+TE}$.

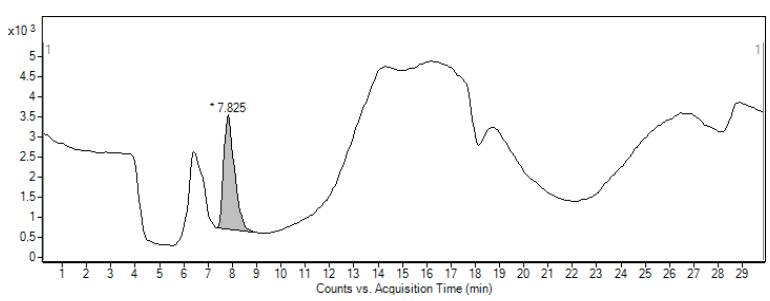

(b) $\left(\right.$ Amp KR $\left.\mathrm{KR}_{2}\right) \mathrm{LipPks1+TE}$.

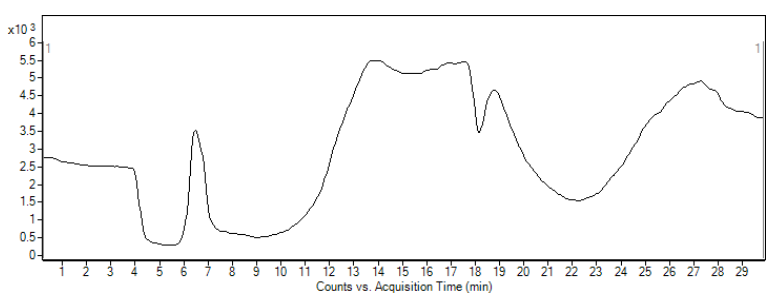

(d) $\left(\right.$ Con $\left.\mathrm{KR}_{2}\right) \mathrm{LipPks} 1+\mathrm{TE}$.

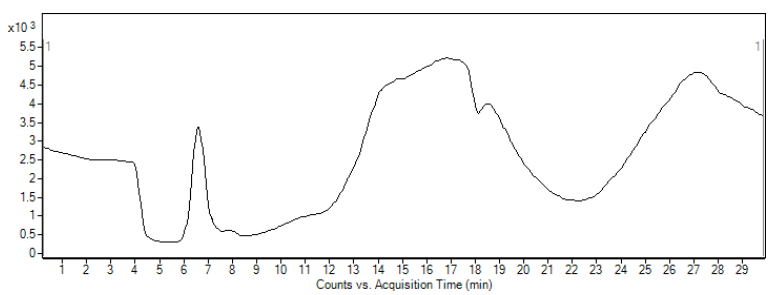

(f) $\left(\right.$ Ery $\left.\mathrm{KR}_{1}\right) \operatorname{LipPks1+TE}$.

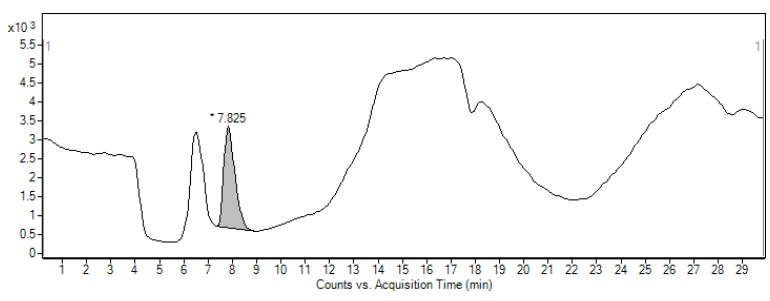

(h) $\left(\mathrm{Spn} \mathrm{KR}_{3}\right) \mathrm{LipPks1+TE}$.

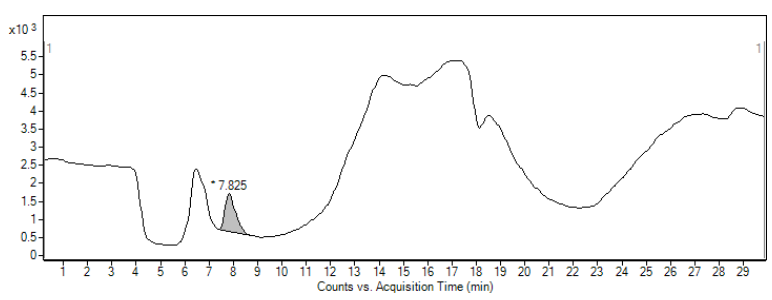

(j) (Ery $\left.\mathrm{KR}_{6}\right) \mathrm{LipPks1+TE}$.

Figure 6: Overnight production of $\mathbf{3 b}$. 


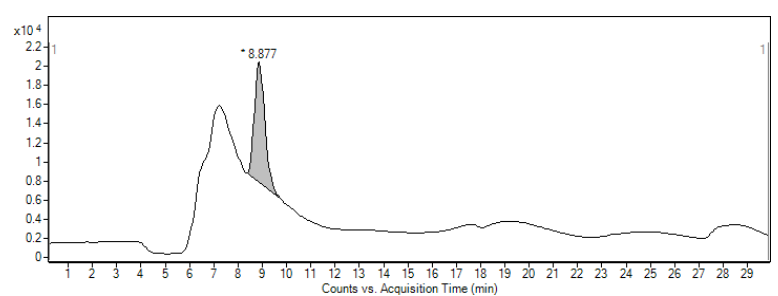

(a) Wild-type LipPks1+TE.

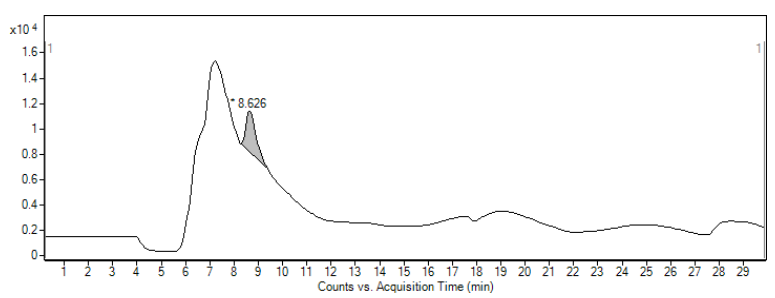

(c) (Amp DE,KR 2$) \mathrm{LipPks1+TE.}$

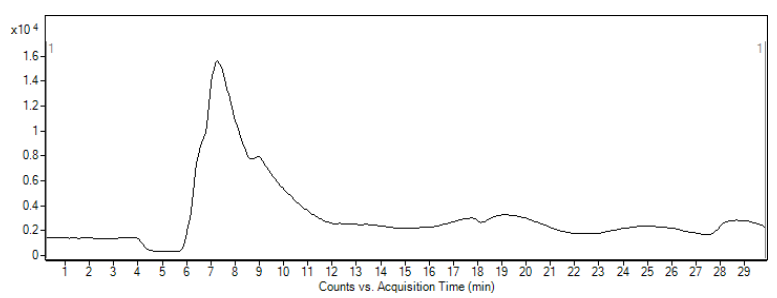

(e) (Con DE,KR 2 LipPks1+TE.

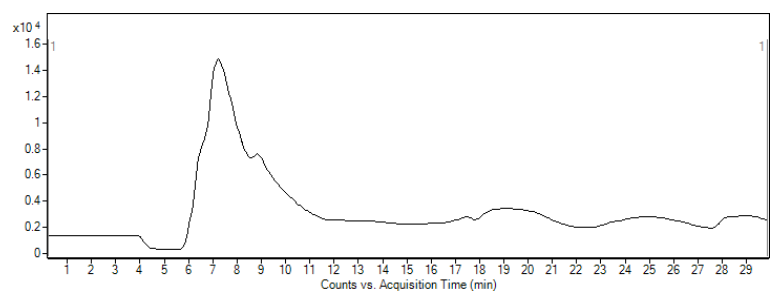

(g) $\left(\right.$ Bor $\left.\mathrm{KR}_{1}\right) \mathrm{LipPks1+TE}$.

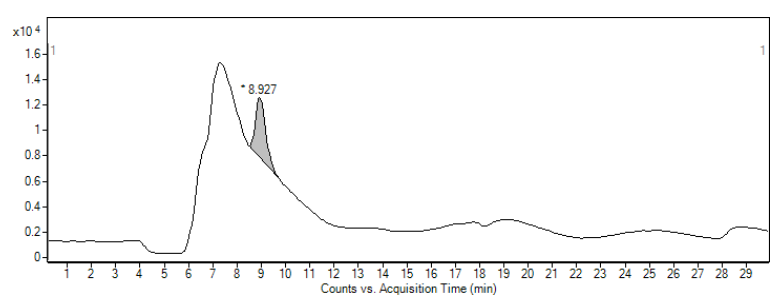

(i) $\left(\operatorname{Amp} \mathrm{KR}_{1}\right) \mathrm{LipPks1+TE}$.

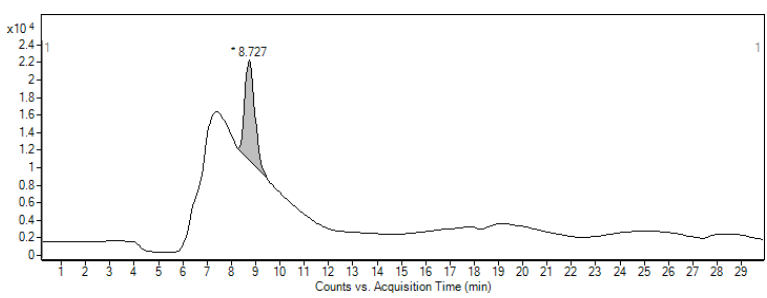

(b) $\left(\right.$ Amp KR $\left.\mathrm{KR}_{2}\right) \mathrm{LipPks1+TE}$.

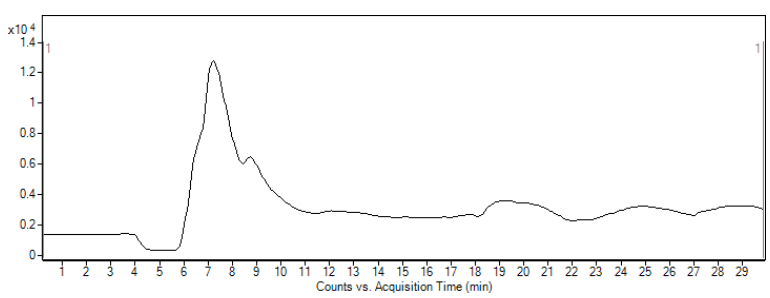

(d) $\left(\right.$ Con $\left.\mathrm{KR}_{2}\right) \mathrm{LipPks} 1+\mathrm{TE}$.

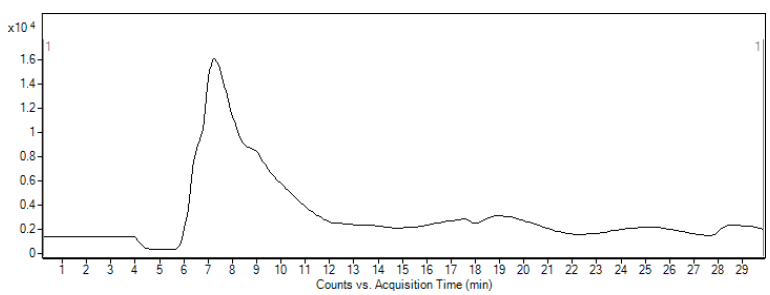

(f) $\left(\right.$ Ery $\left.\mathrm{KR}_{1}\right) \mathrm{LipPks1}+\mathrm{TE}$.

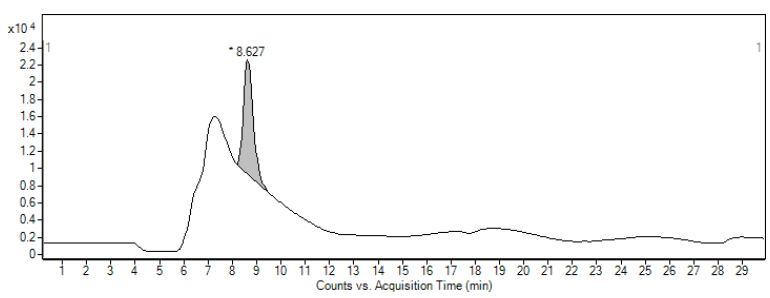

(h) $\left(\mathrm{Spn} \mathrm{KR}_{3}\right) \mathrm{LipPks1+TE}$.

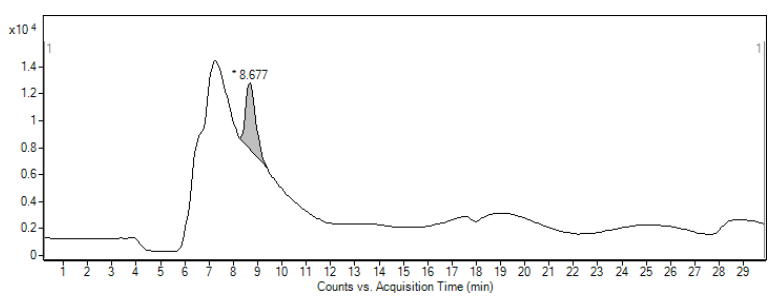

(j) (Ery $\left.\mathrm{KR}_{6}\right) \mathrm{LipPks1+TE}$.

Figure 7: Overnight production of 3c. 


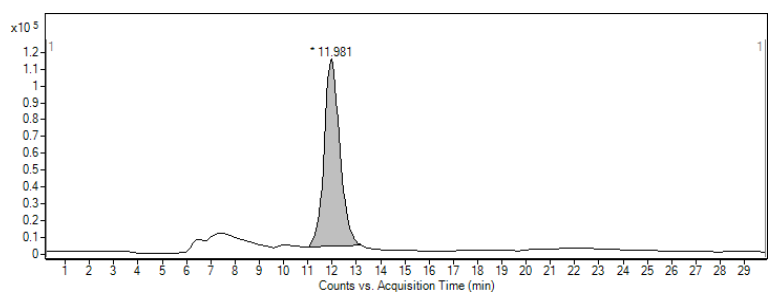

(a) Wild-type LipPks1+TE.

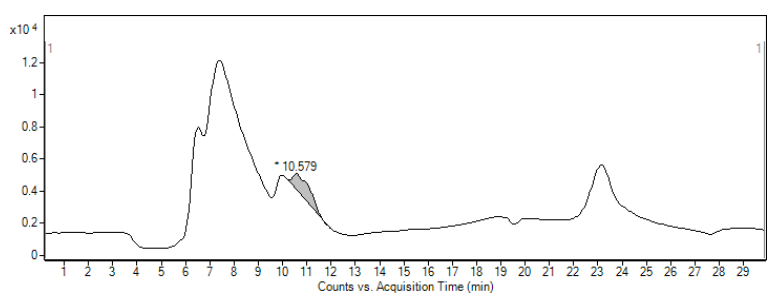

(c) $\left(\mathrm{Amp} \mathrm{DE}, \mathrm{KR}_{2}\right) \mathrm{LipPks} 1+\mathrm{TE}$.

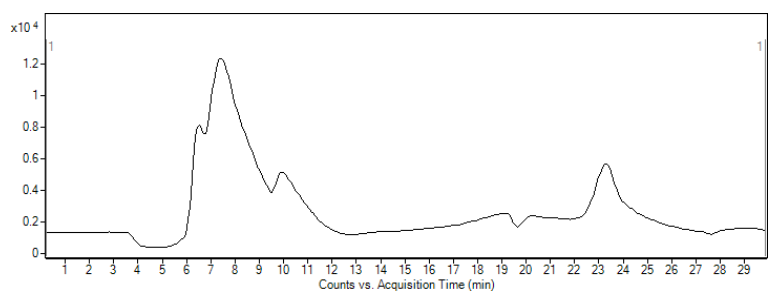

(e) (Con DE,KR 2 LipPks1+TE.

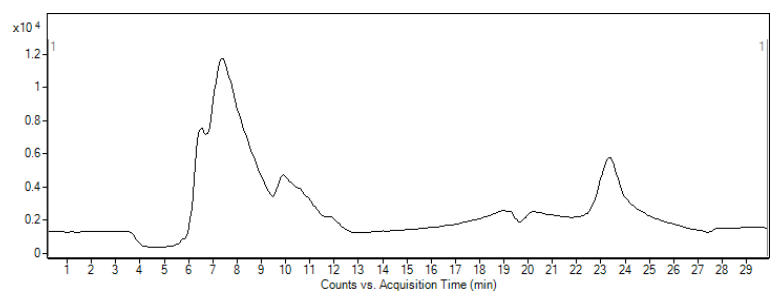

(g) $\left(\right.$ Bor $\left.\mathrm{KR}_{1}\right) \mathrm{LipPks1+TE}$.

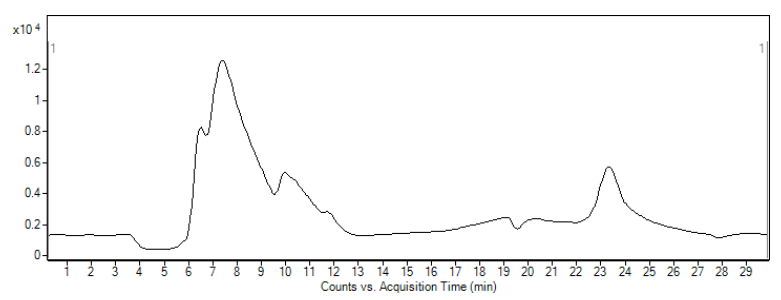

(i) $\left(\operatorname{Amp} \mathrm{KR}_{1}\right) \mathrm{LipPks1+TE}$.

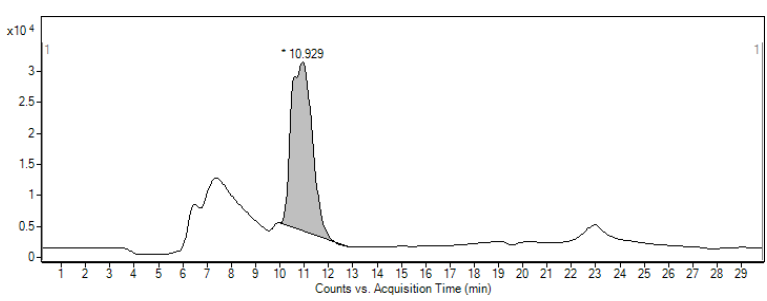

(b) $\left(\right.$ Amp KR $\left.\mathrm{KR}_{2}\right) \mathrm{LipPks1+TE}$.

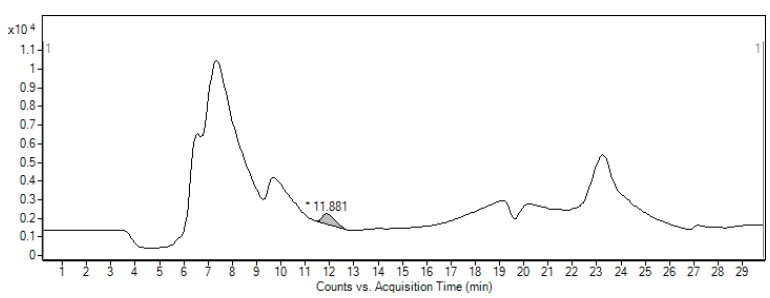

(d) $\left(\right.$ Con $\left.\mathrm{KR}_{2}\right) \mathrm{LipPks} 1+\mathrm{TE}$.

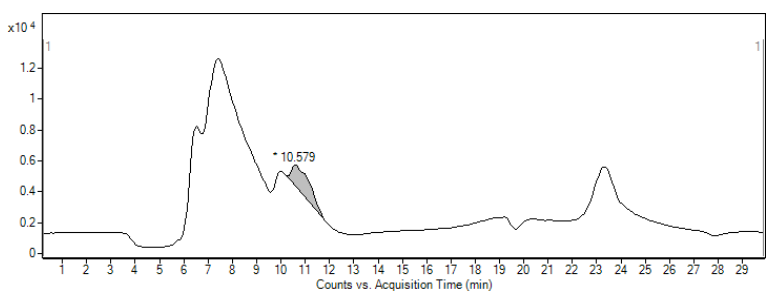

(f) $\left(\right.$ Ery $\left.\mathrm{KR}_{1}\right) \operatorname{LipPks1+TE}$.

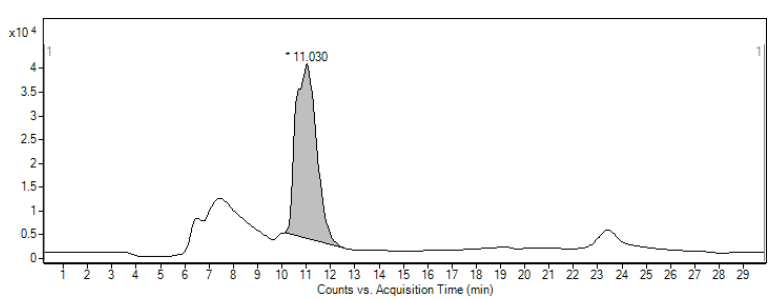

(h) $\left(\mathrm{Spn} \mathrm{KR}_{3}\right) \mathrm{LipPks1+TE}$.

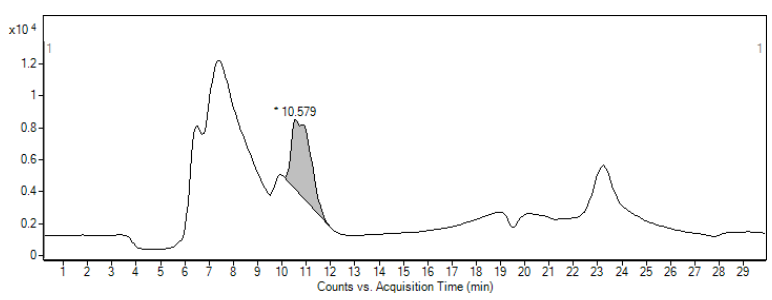

(j) (Ery $\left.\mathrm{KR}_{6}\right) \mathrm{LipPks1+TE}$.

Figure 8: Overnight production of $\mathbf{3 d}$. 


\section{Production of 2a-d by LipPks1+TE Variants (LC-MS Chro- matograms)}

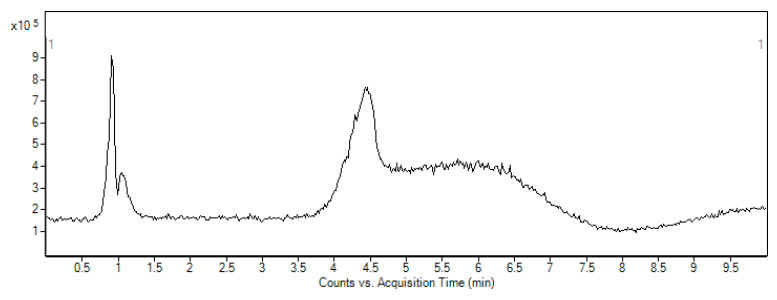

(a) $0 \mu \mathrm{M} \mathbf{2 a}$.

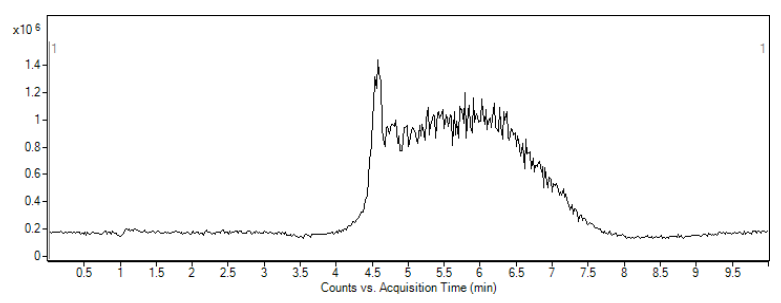

(c) $0 \mu \mathrm{M} \mathbf{2} \mathbf{b}$.

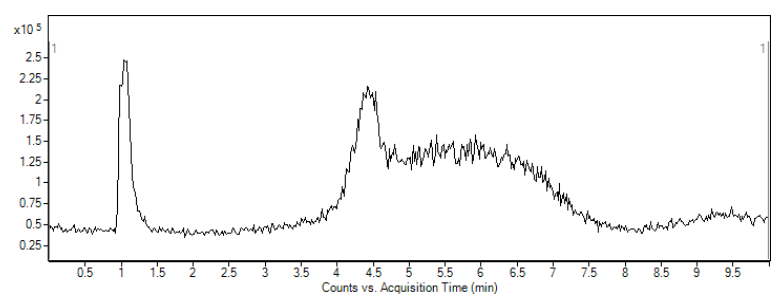

(e) $0 \mu \mathrm{M} \mathbf{2 c}$.

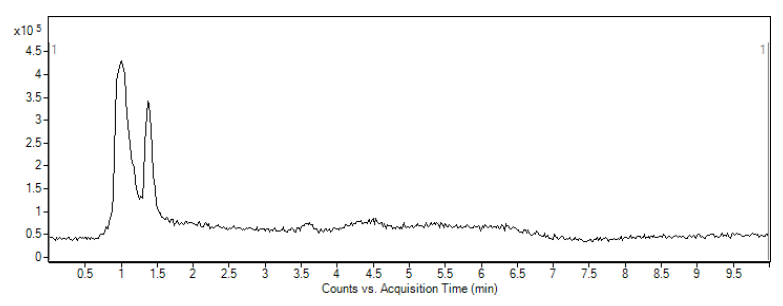

(g) $0 \mu \mathrm{M} \mathbf{2 d}$.

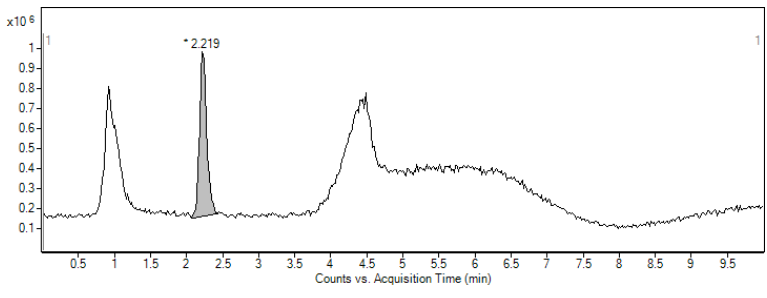

(b) $25 \mu \mathrm{M} 2 \mathrm{a}$.

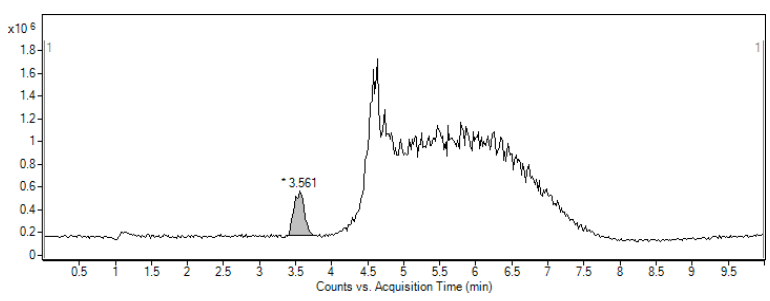

(d) $25 \mu \mathrm{M} \mathbf{2 b}$.

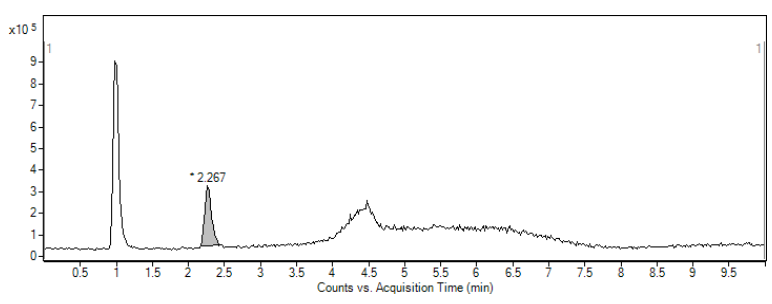

(f) $25 \mu \mathrm{M} 2 \mathbf{c}$.

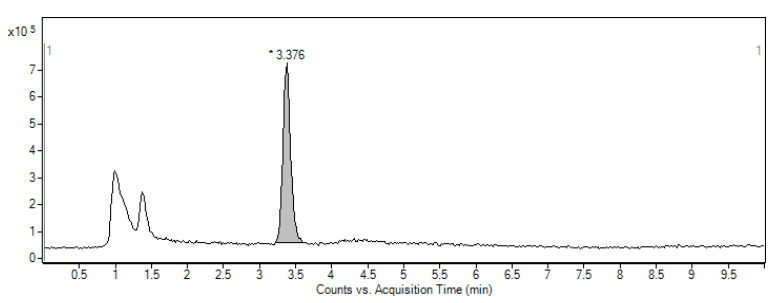

(h) $25 \mu \mathrm{M} \mathbf{2 d}$.

Figure 9: Authentic ketone standards 2a-d. 


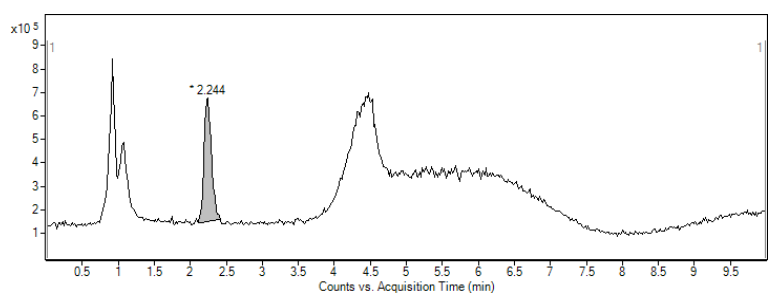

(a) Wild-type LipPks1+TE.

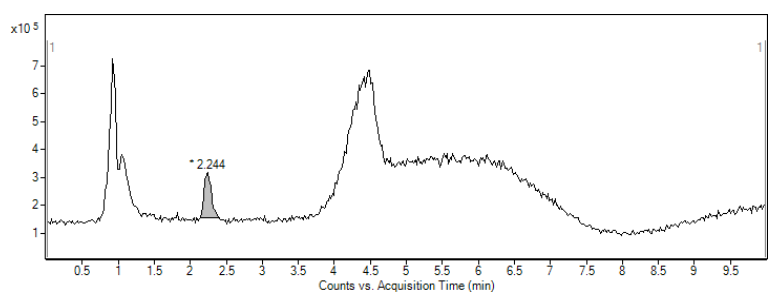

(c) $\left(\mathrm{Amp} \mathrm{DE}, \mathrm{KR}_{2}\right) \mathrm{LipPks} 1+\mathrm{TE}$.

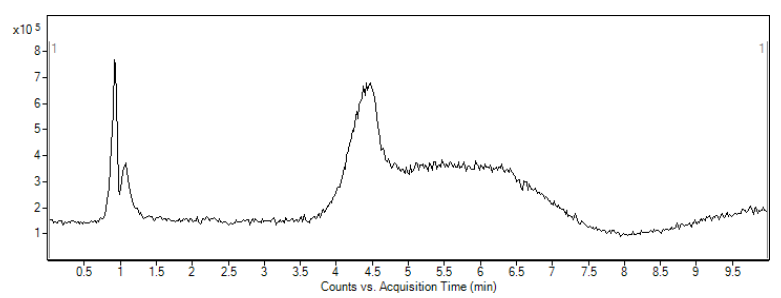

(e) (Con DE,KR 2 LipPks1+TE.

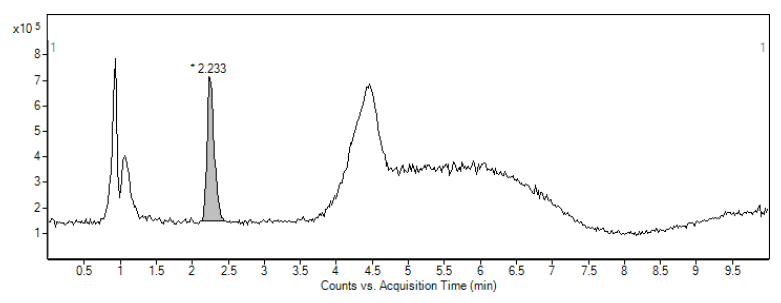

(g) $\left(\right.$ Bor $\left.\mathrm{KR}_{1}\right) \operatorname{LipPks1+TE}$.

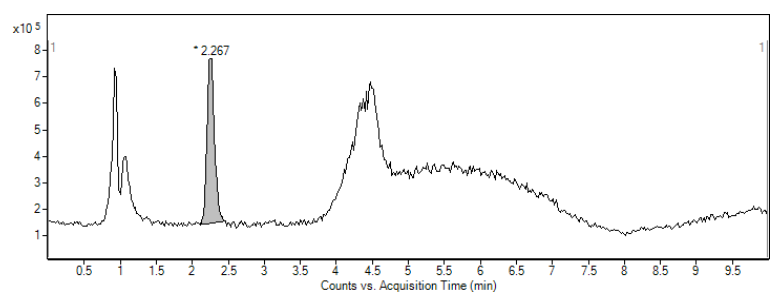

(i) $\left(\operatorname{Amp} \mathrm{KR}_{1}\right) \mathrm{LipPks1+TE}$.

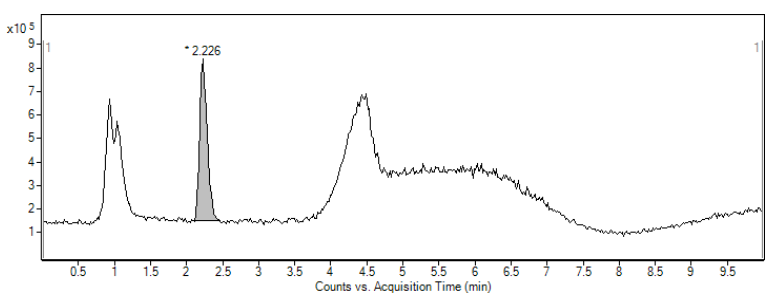

(b) $\left(\right.$ Amp KR $\left.\mathrm{KR}_{2}\right) \mathrm{LipPks1+TE}$.

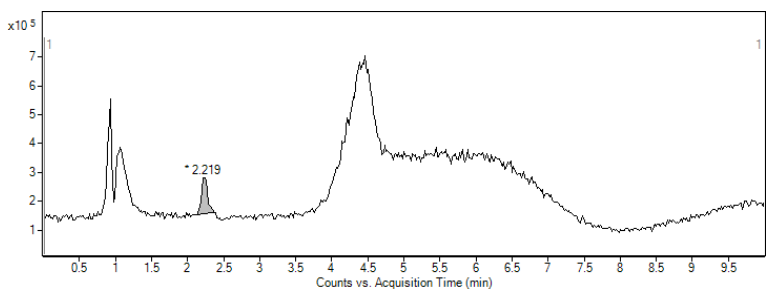

(d) $\left(\right.$ Con $\left.\mathrm{KR}_{2}\right) \mathrm{LipPks} 1+\mathrm{TE}$.

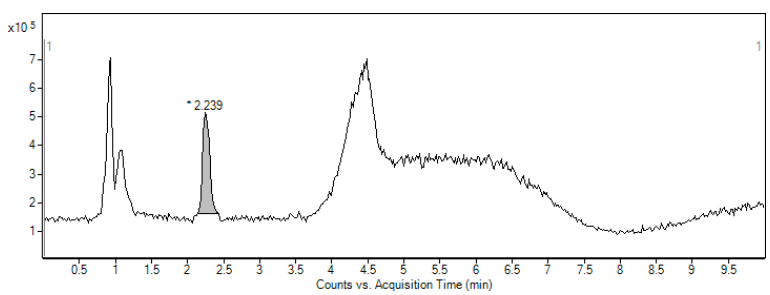

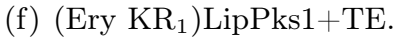

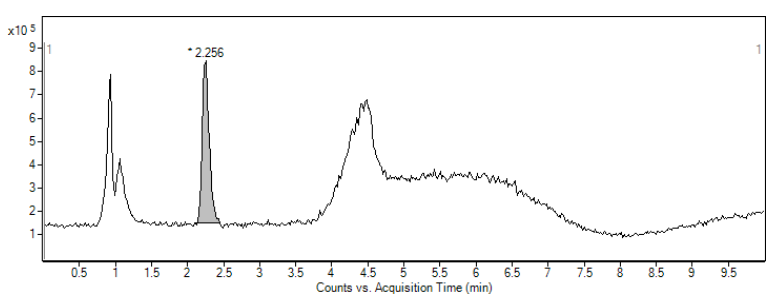

(h) $\left(\mathrm{Spn} \mathrm{KR}_{3}\right) \mathrm{LipPks1+TE}$.

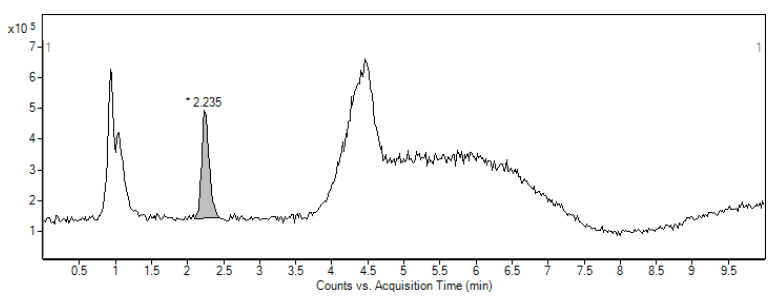

(j) (Ery $\left.\mathrm{KR}_{6}\right) \mathrm{LipPks1+TE}$.

Figure 10: Overnight production of $\mathbf{2 a}$. 


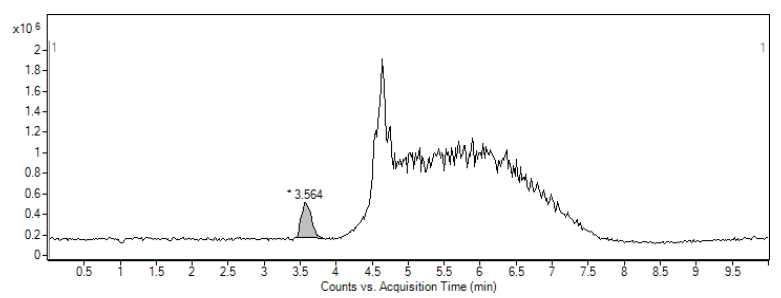

(a) Wild-type LipPks1+TE.

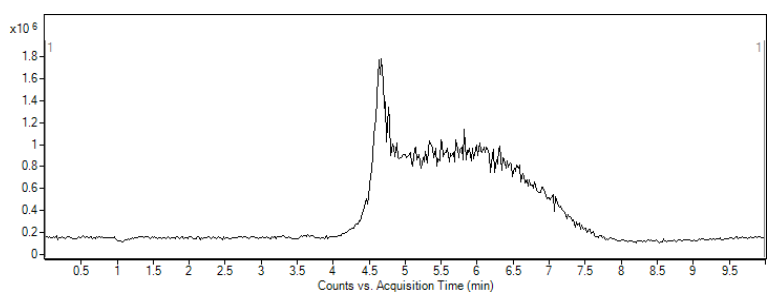

(c) $\left(\mathrm{Amp} \mathrm{DE}, \mathrm{KR}_{2}\right) \mathrm{LipPks} 1+\mathrm{TE}$.

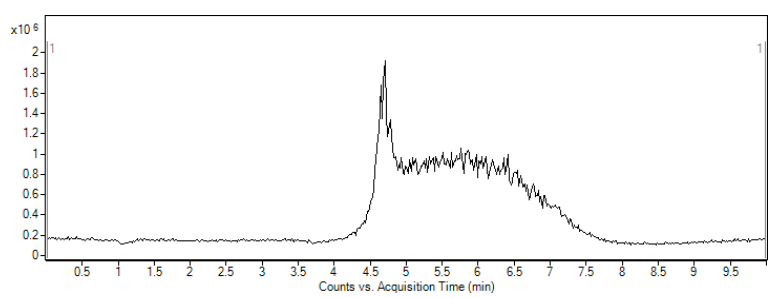

(e) (Con DE,KR 2 LipPks1+TE.

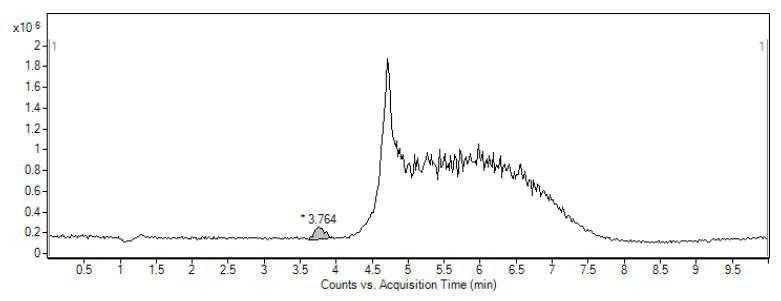

(g) $\left(\right.$ Bor $\left.\mathrm{KR}_{1}\right) \mathrm{LipPks1+TE}$.

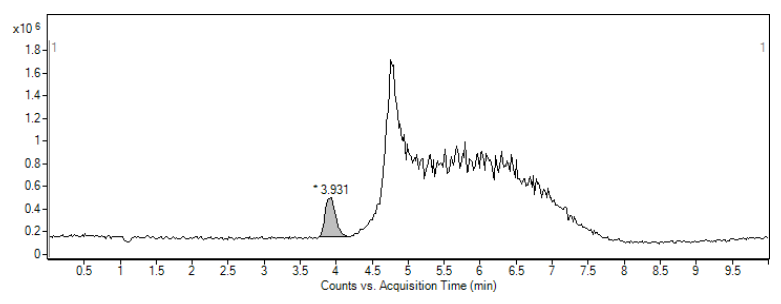

(i) $\left(\operatorname{Amp} \mathrm{KR}_{1}\right) \mathrm{LipPks1+TE}$.

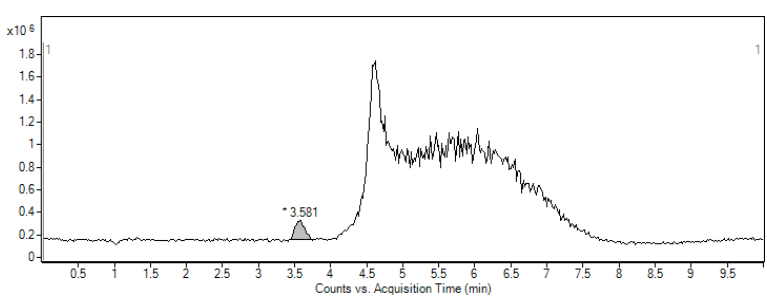

(b) $(\mathrm{Amp} \mathrm{KR} 2) \mathrm{LipPks1+TE}$.

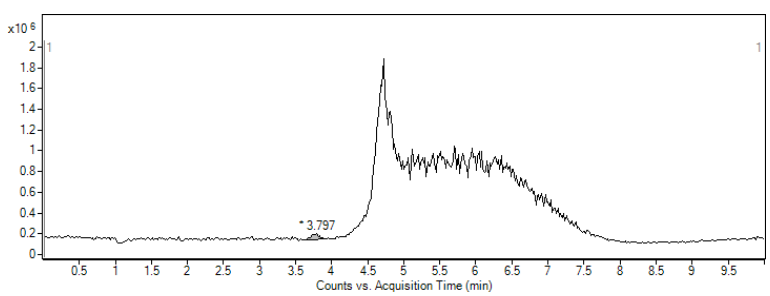

(d) $\left(\right.$ Con $\left.\mathrm{KR}_{2}\right) \mathrm{LipPks} 1+\mathrm{TE}$.

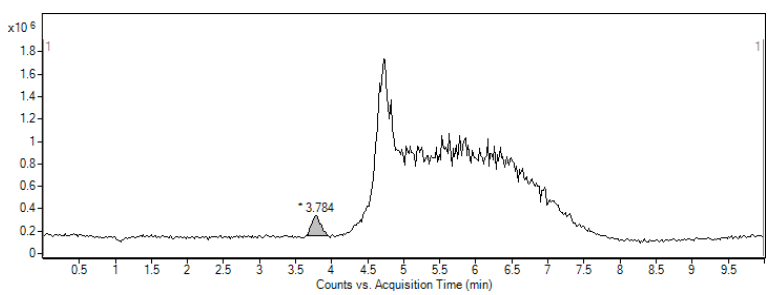

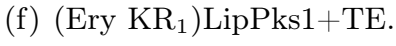

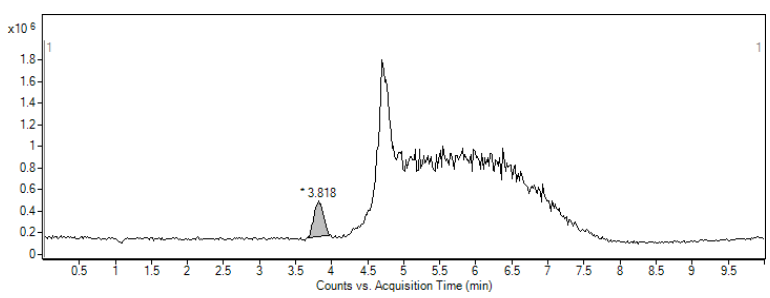

(h) $\left(\mathrm{Spn} \mathrm{KR}_{3}\right) \mathrm{LipPks} 1+\mathrm{TE}$.

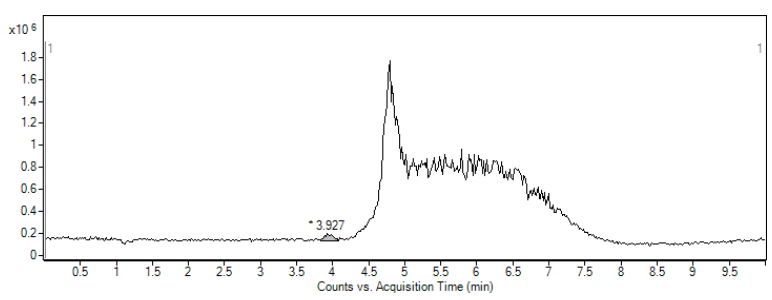

(j) (Ery $\left.\mathrm{KR}_{6}\right) \mathrm{LipPks1+TE}$.

Figure 11: Overnight production of $\mathbf{2 b}$. 


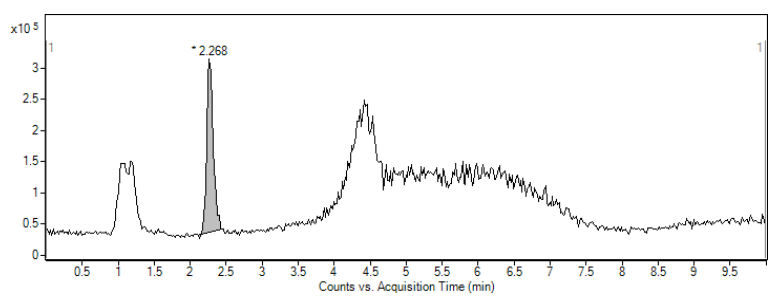

(a) Wild-type LipPks1+TE.

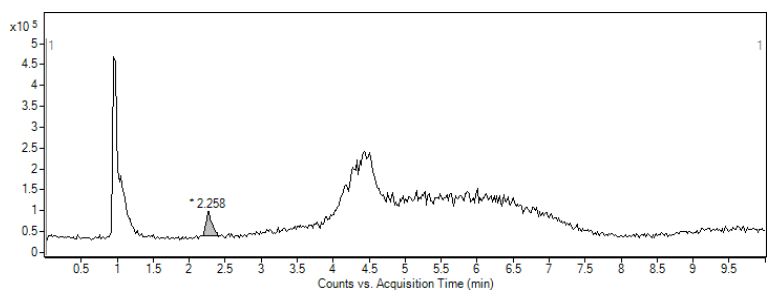

(c) (Amp DE,KR 2$) \mathrm{LipPks1+TE.}$

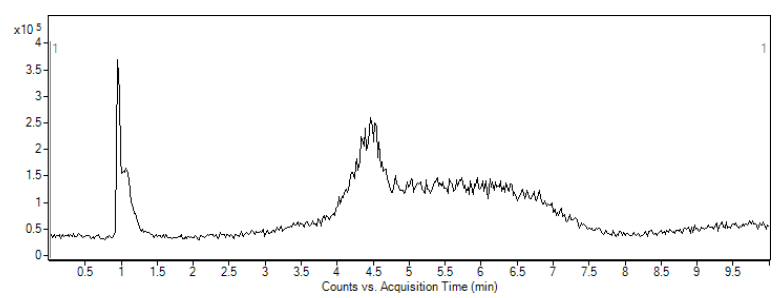

(e) (Con DE,KR 2 LipPks1+TE.

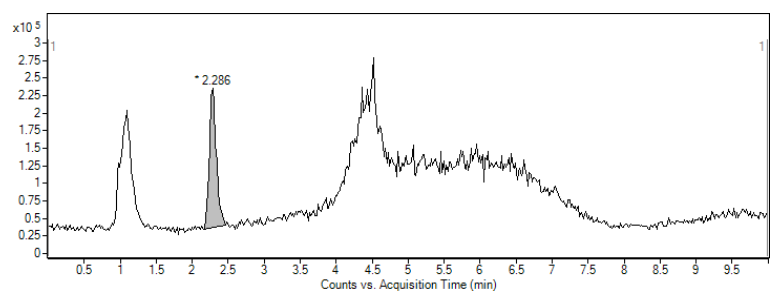

(g) $\left(\right.$ Bor $\left.\mathrm{KR}_{1}\right) \mathrm{LipPks1+TE}$.

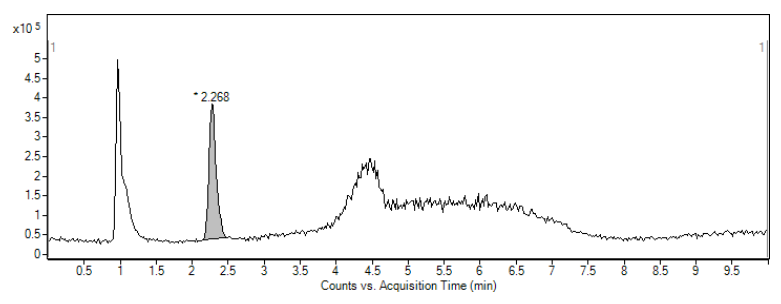

(i) $\left(\operatorname{Amp} \mathrm{KR}_{1}\right) \mathrm{LipPks1+TE}$.

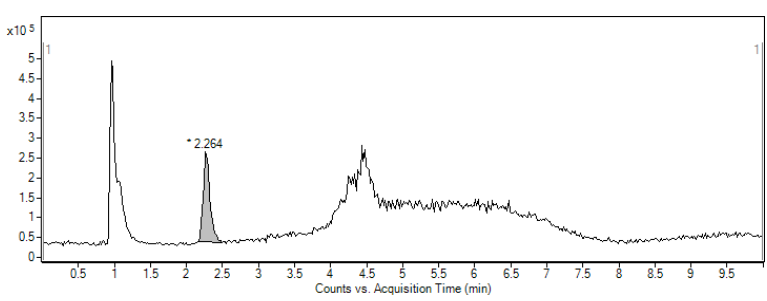

(b) $\left(\right.$ Amp KR $\left.\mathrm{KR}_{2}\right) \mathrm{LipPks1+TE}$.

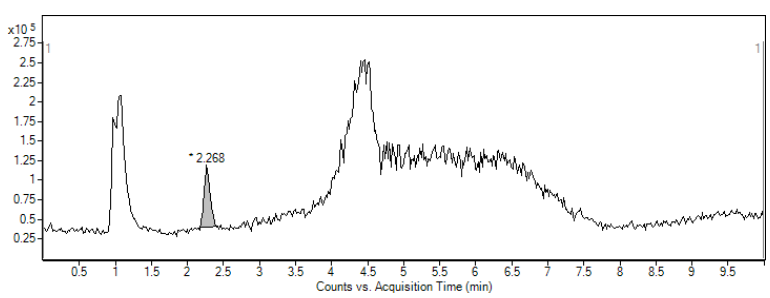

(d) $\left(\right.$ Con $\left.\mathrm{KR}_{2}\right) \mathrm{LipPks} 1+\mathrm{TE}$.

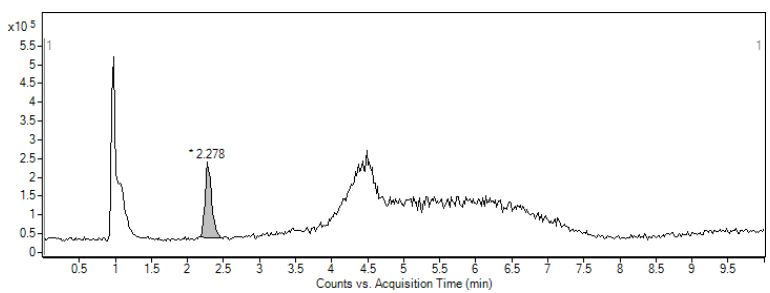

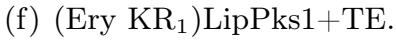

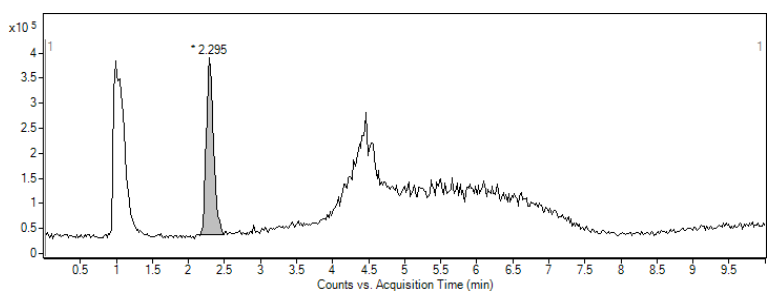

(h) $\left(\mathrm{Spn} \mathrm{KR}_{3}\right) \mathrm{LipPks} 1+\mathrm{TE}$.

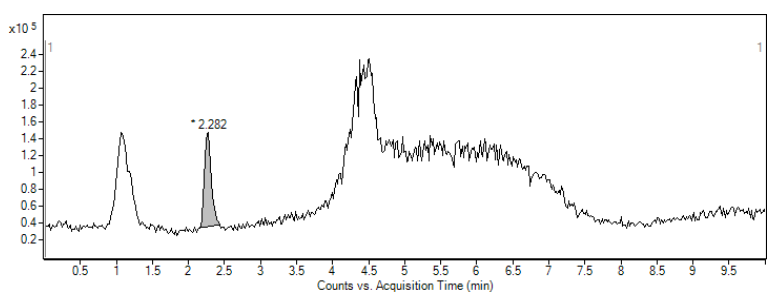

(j) (Ery $\left.\mathrm{KR}_{6}\right) \mathrm{LipPks1+TE}$.

Figure 12: Overnight production of 2c. 


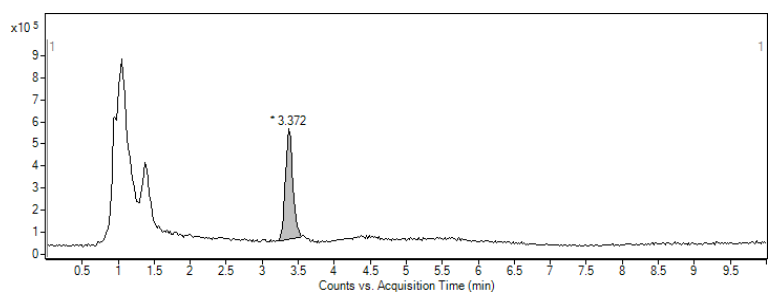

(a) Wild-type LipPks1+TE.

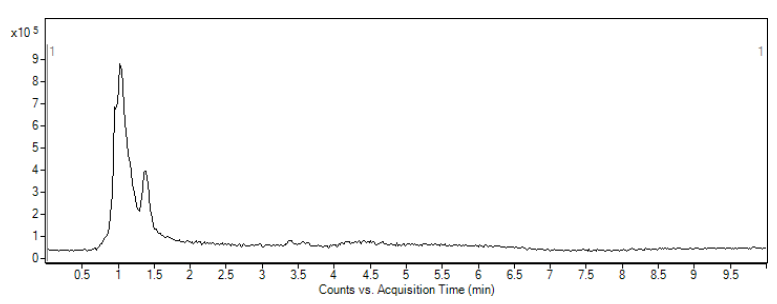

(c) (Amp DE,KR 2$) \mathrm{LipPks} 1+\mathrm{TE}$.

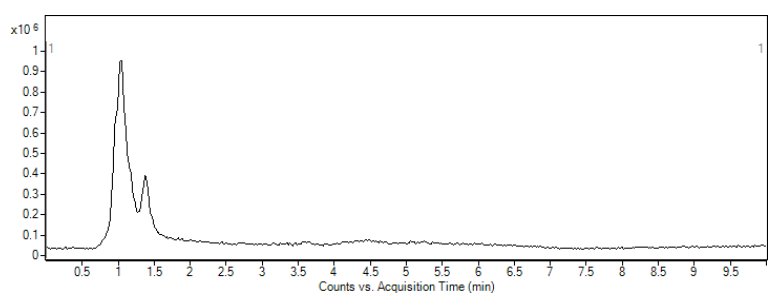

(e) (Con DE,KR 2 LipPks1+TE.

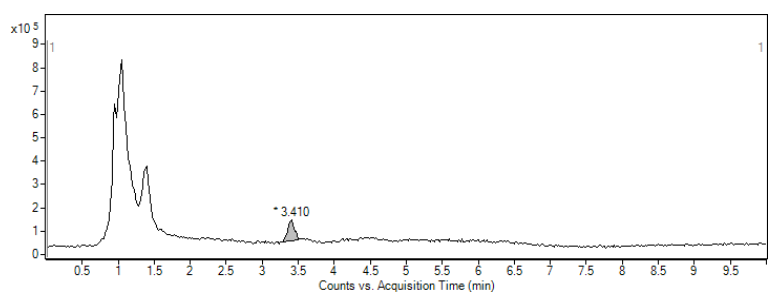

(g) $\left(\right.$ Bor $\left.\mathrm{KR}_{1}\right) \mathrm{LipPks} 1+\mathrm{TE}$.

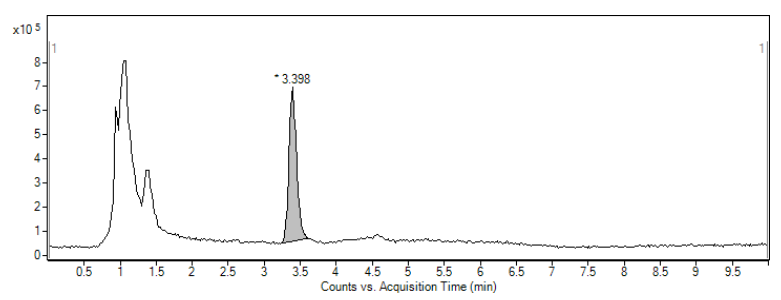

(i) $\left(\operatorname{Amp} \mathrm{KR}_{1}\right) \mathrm{LipPks} 1+\mathrm{TE}$.

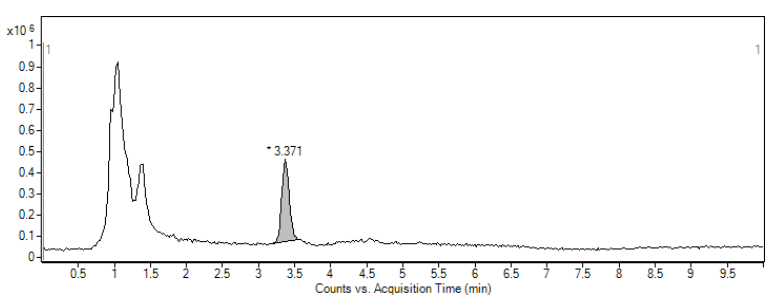

(b) $\left(\right.$ Amp KR $\left.\mathrm{KR}_{2}\right) \mathrm{LipPks1+TE}$.

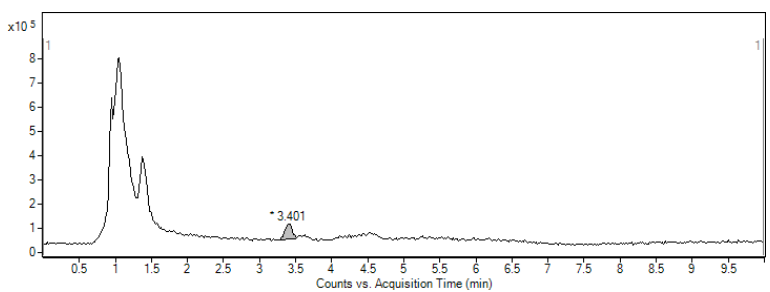

(d) $\left(\right.$ Con $\left.\mathrm{KR}_{2}\right) \mathrm{LipPks} 1+\mathrm{TE}$.

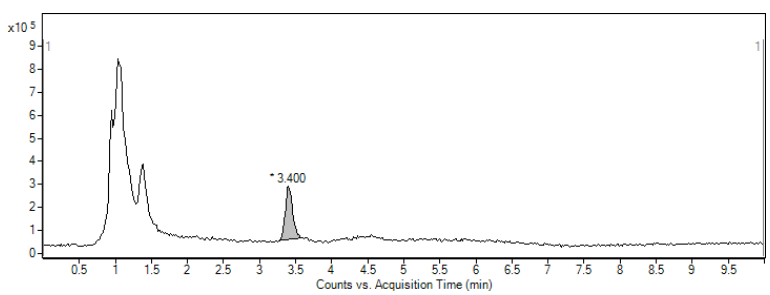

(f) $\left(\right.$ Ery $\left.\mathrm{KR}_{1}\right) \mathrm{LipPks} 1+\mathrm{TE}$.

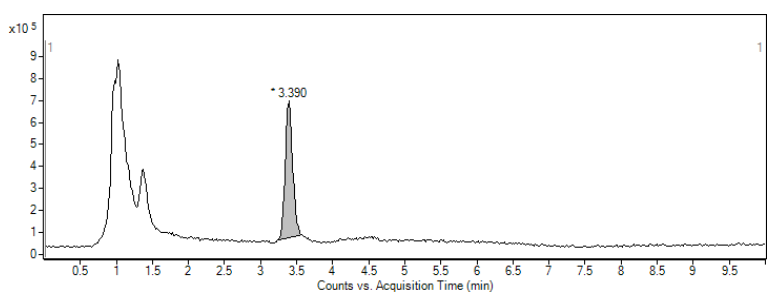

(h) $\left(\mathrm{Spn} \mathrm{KR}_{3}\right) \mathrm{LipPks1+TE}$.

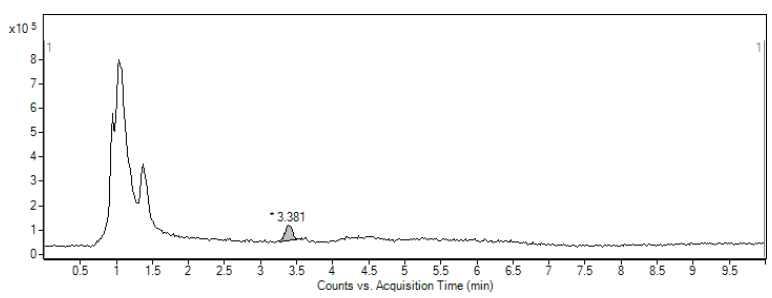

(j) (Ery $\left.\mathrm{KR}_{6}\right) \mathrm{LipPks1+TE}$.

Figure 13: Overnight production of $\mathbf{2 d}$. 


\section{Stereochemical Purity of 3a Produced by A-type LipPks1+TE Variants (Chiral GC-MS Chromatograms)}
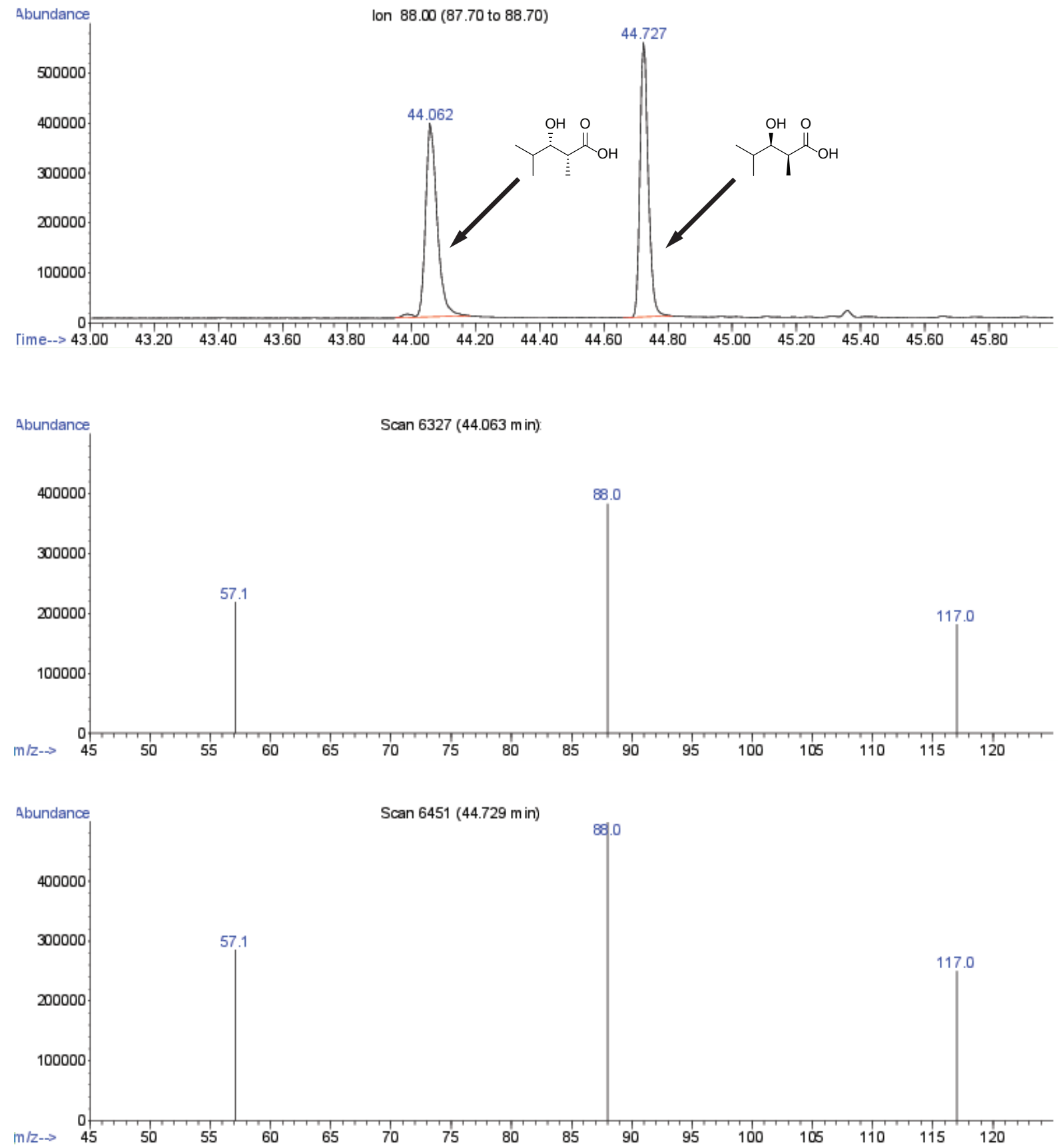

Figure 14: Enantiomeric mixture of synthetic syn standards, $(2 R, 3 S)-\mathbf{3 a}$ and $(2 S, 3 R)-\mathbf{3 a}$, corresponding to A1 and B2 type products. 

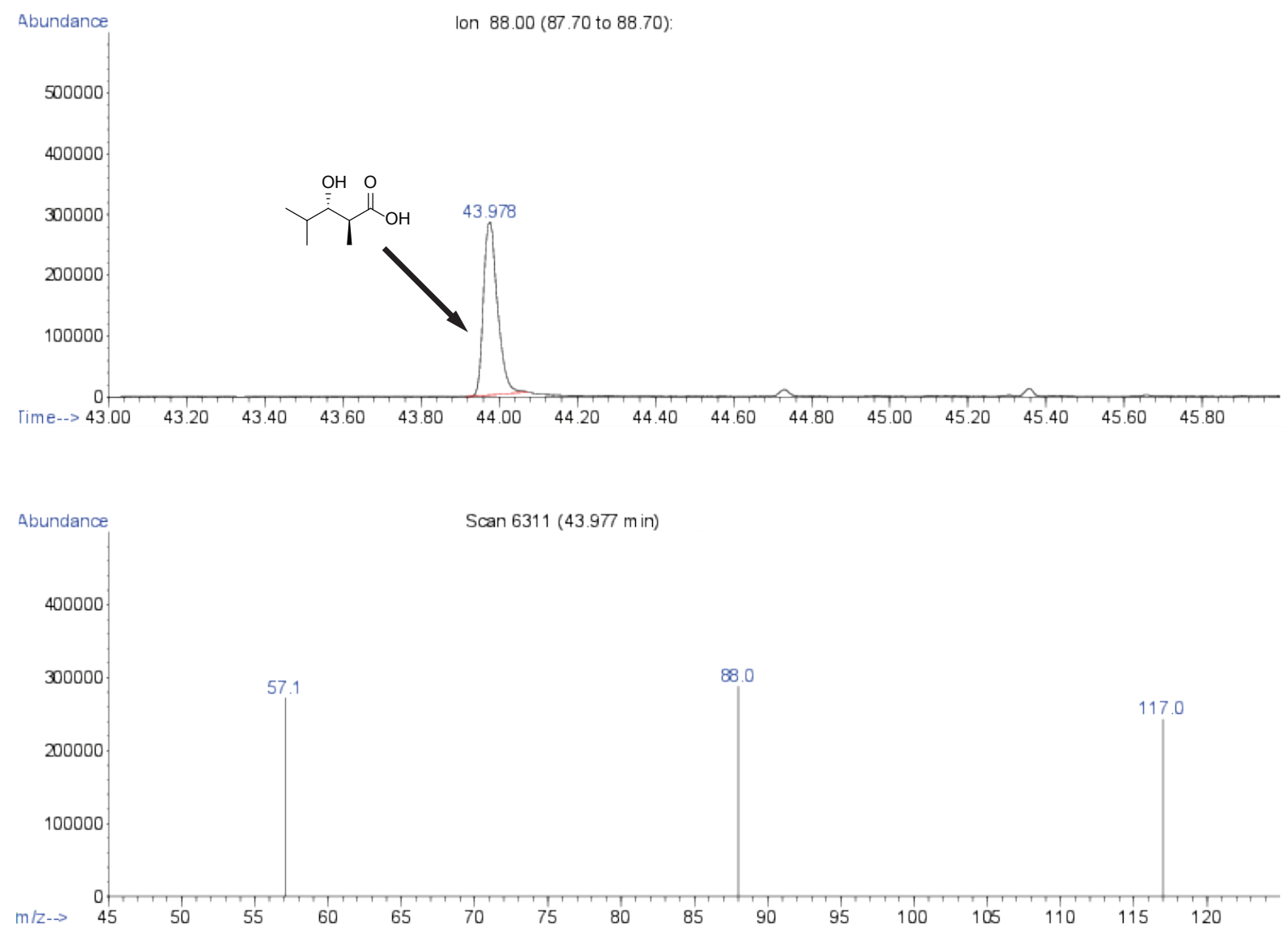

Figure 15: Synthetic (2S,3S)-3a standard corresponding to A2 type product. 

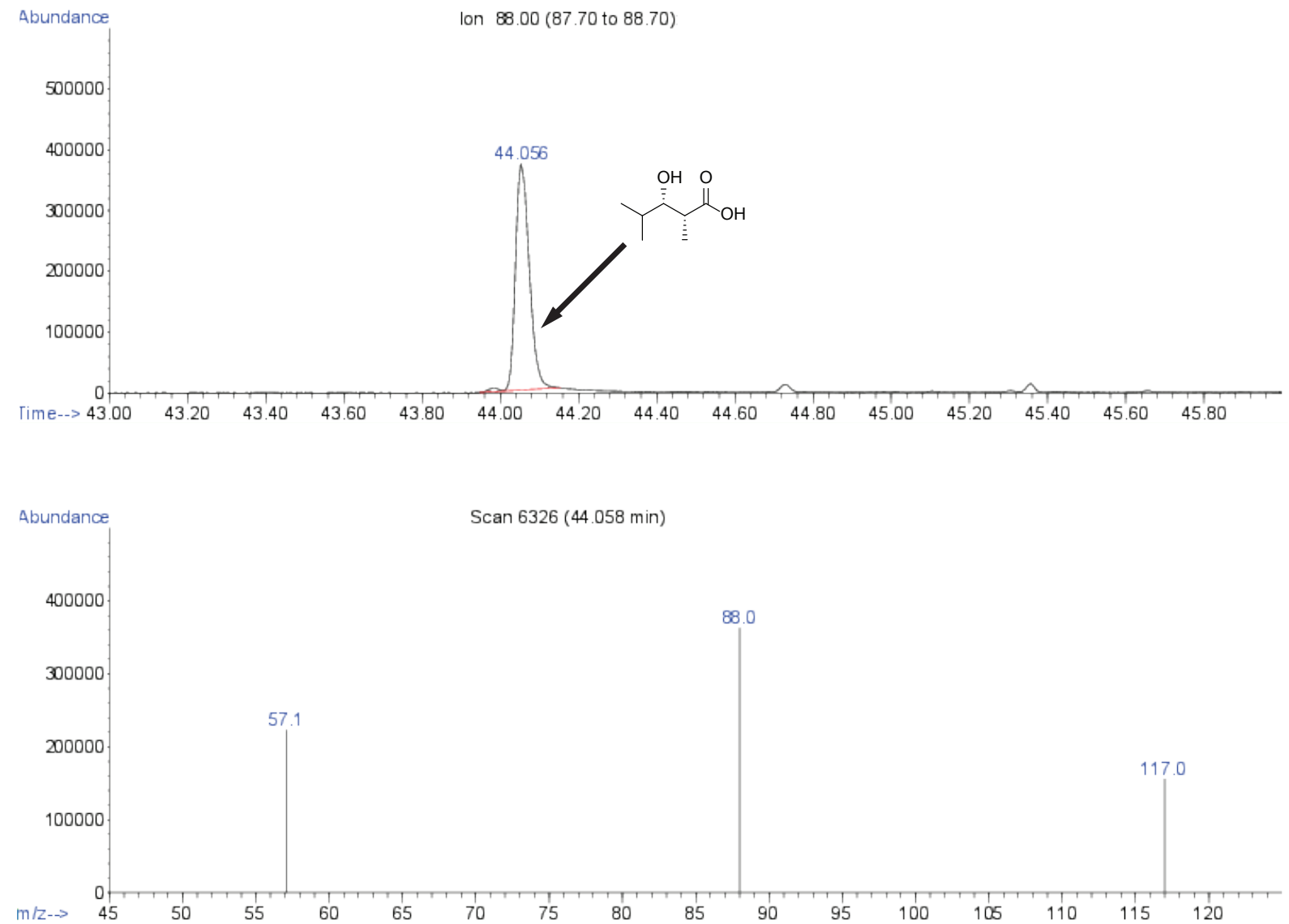

Figure 16: Synthetic (2R,3S)-3a standard corresponding to A1 type product. 

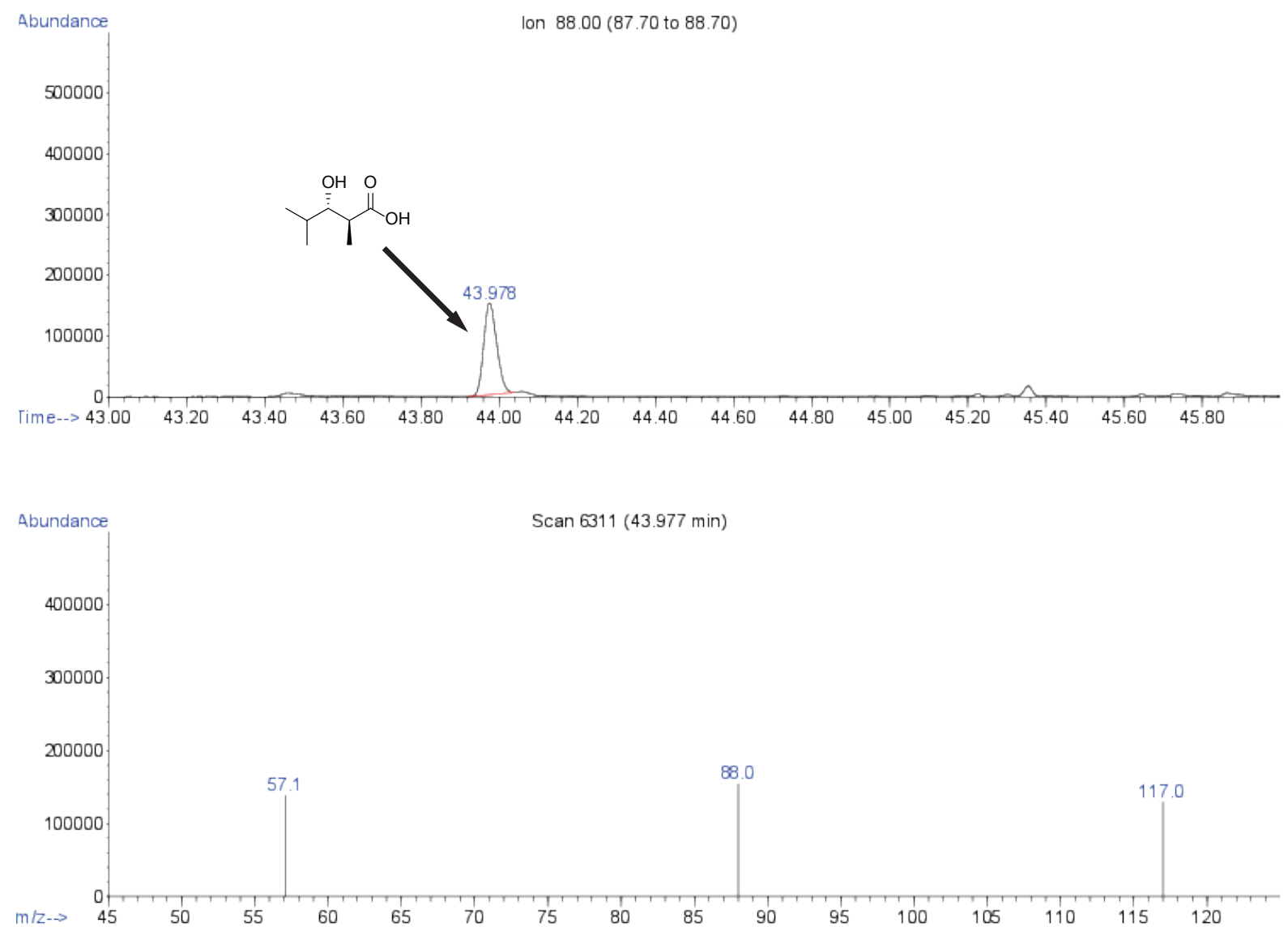

Figure 17: Stereochemical purity of $\mathbf{3 a}$ produced by wild-type LipPks1+TE. 

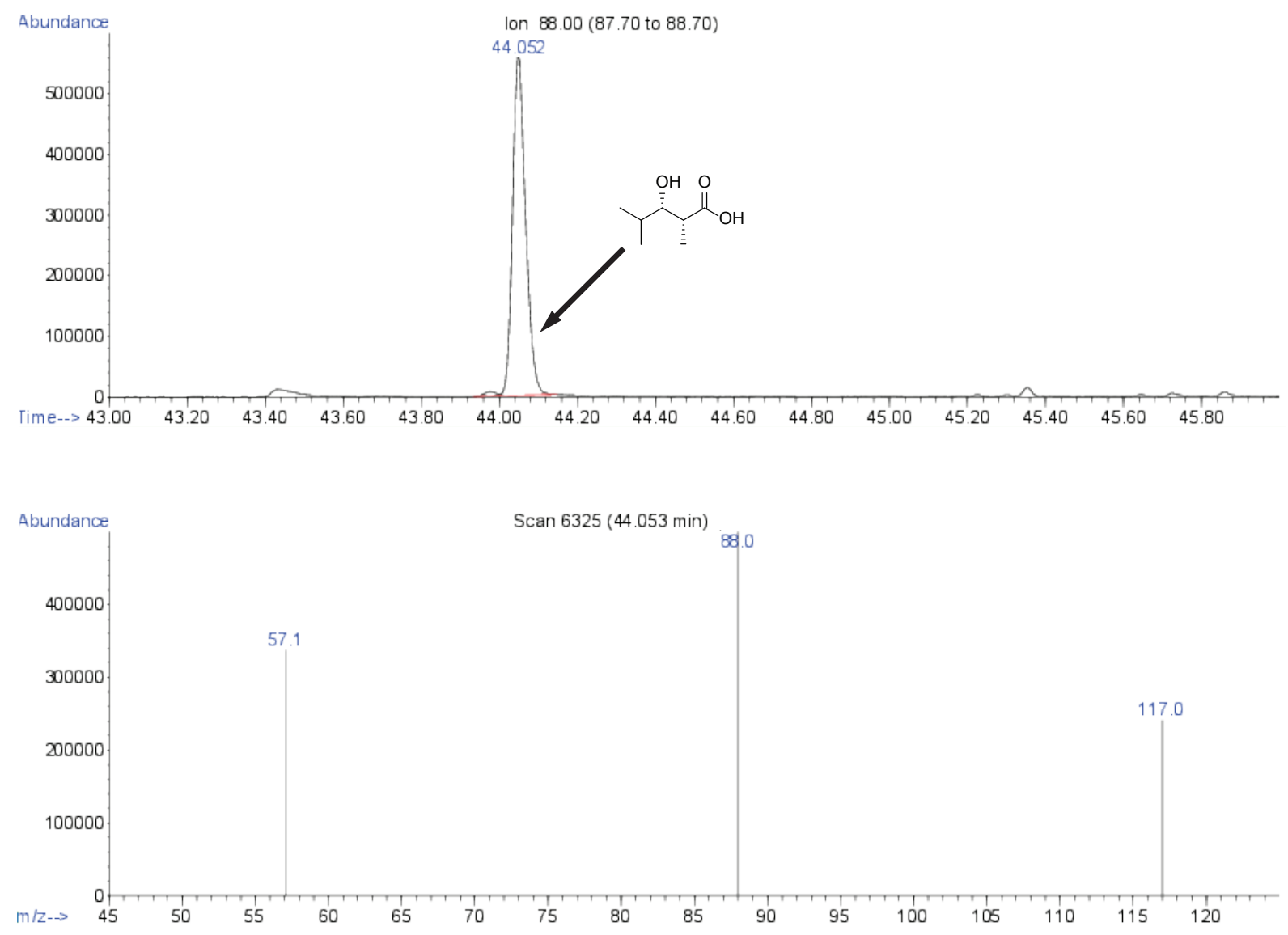

Figure 18: Stereochemical purity of $\mathbf{3 a}$ produced by $\left(\operatorname{Amp} \mathrm{KR}_{2}\right) \operatorname{LipPks1}+\mathrm{TE}$. 

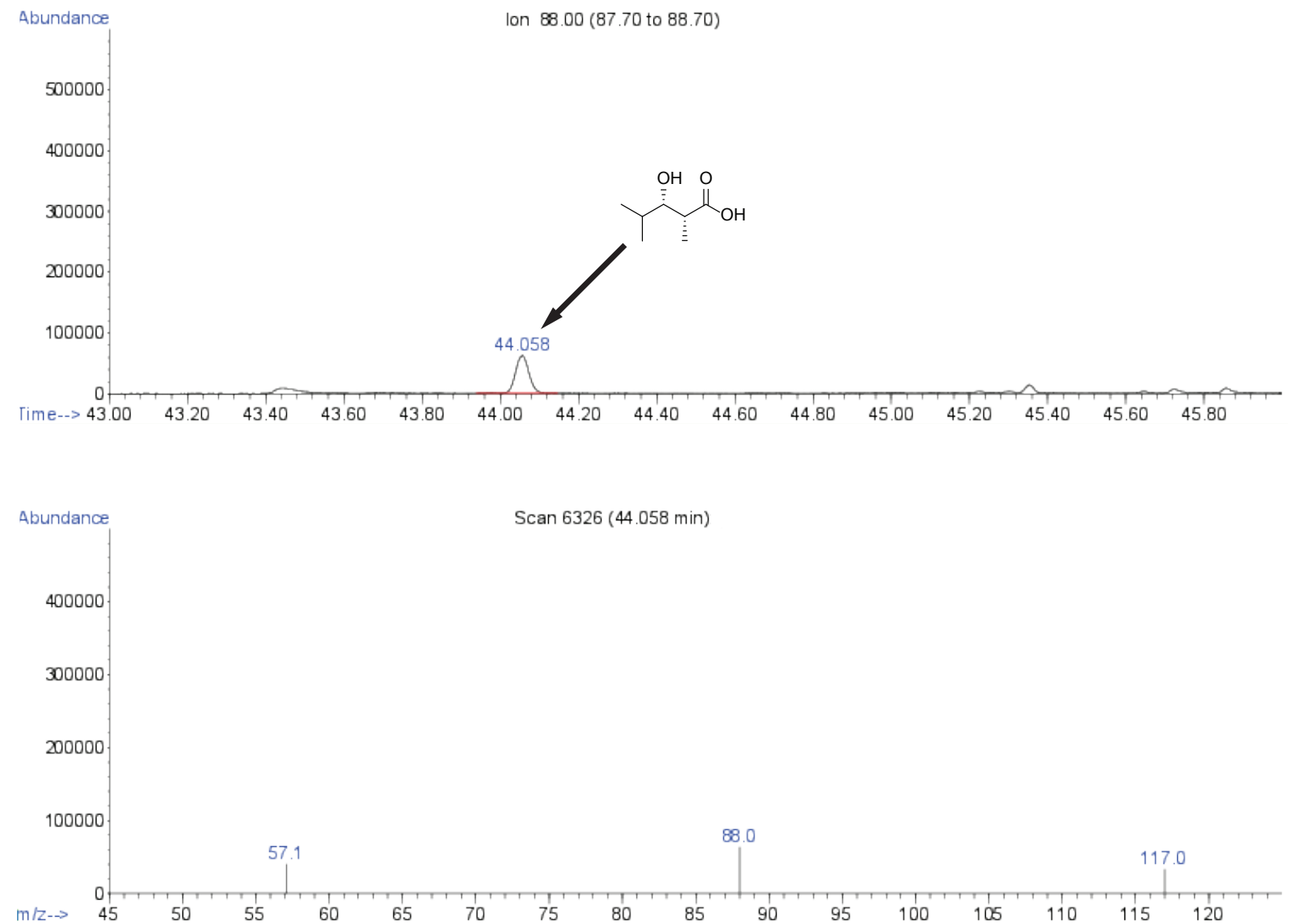

Figure 19: Stereochemical purity of 3a produced by (Amp DE,KR 2$) L i p P k s 1+T E$. 

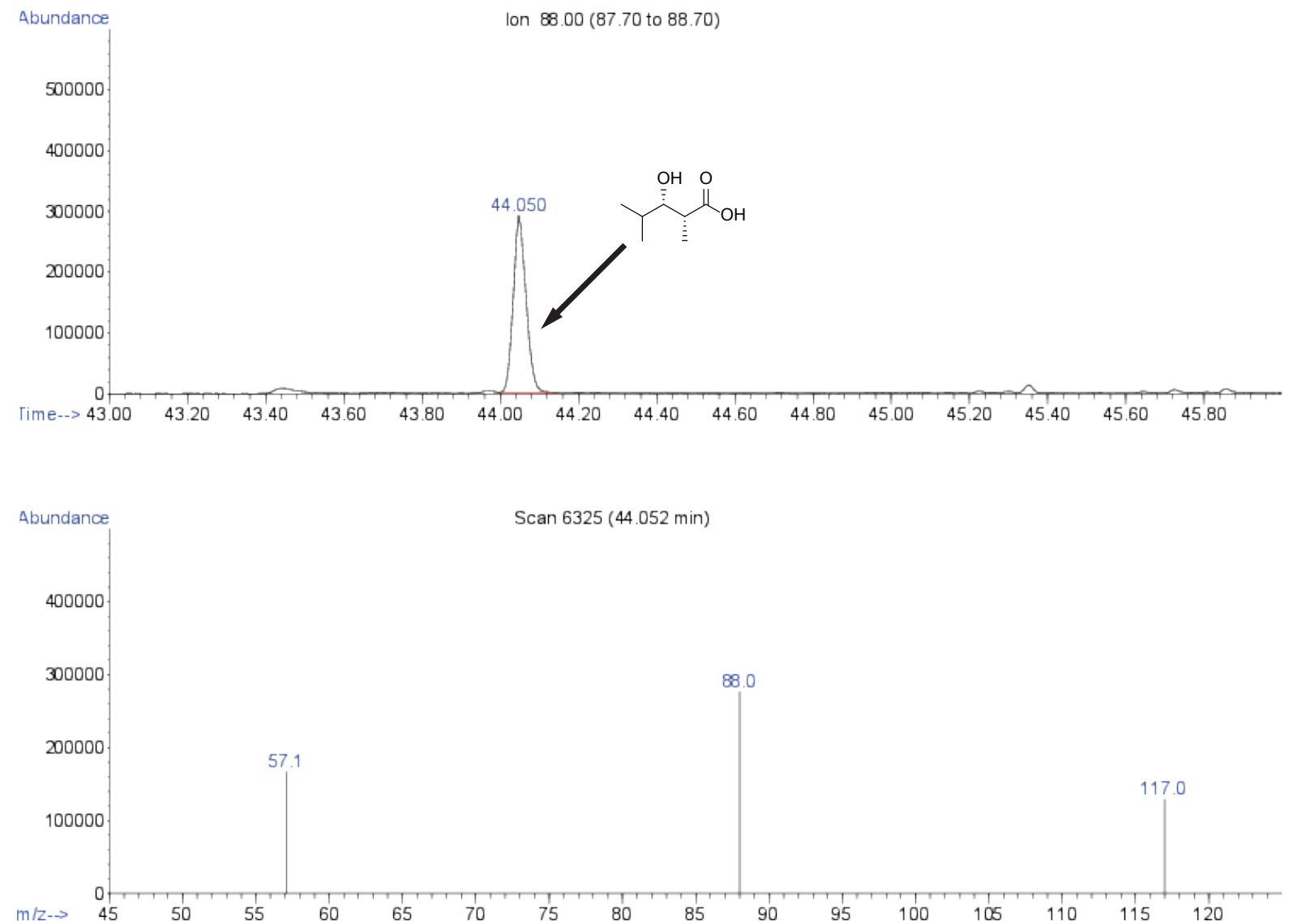

Figure 20: Stereochemical purity of $\mathbf{3 a}$ produced by $\left(\mathrm{Spn} \mathrm{KR}_{3}\right) \mathrm{LipPks1+TE}$. 

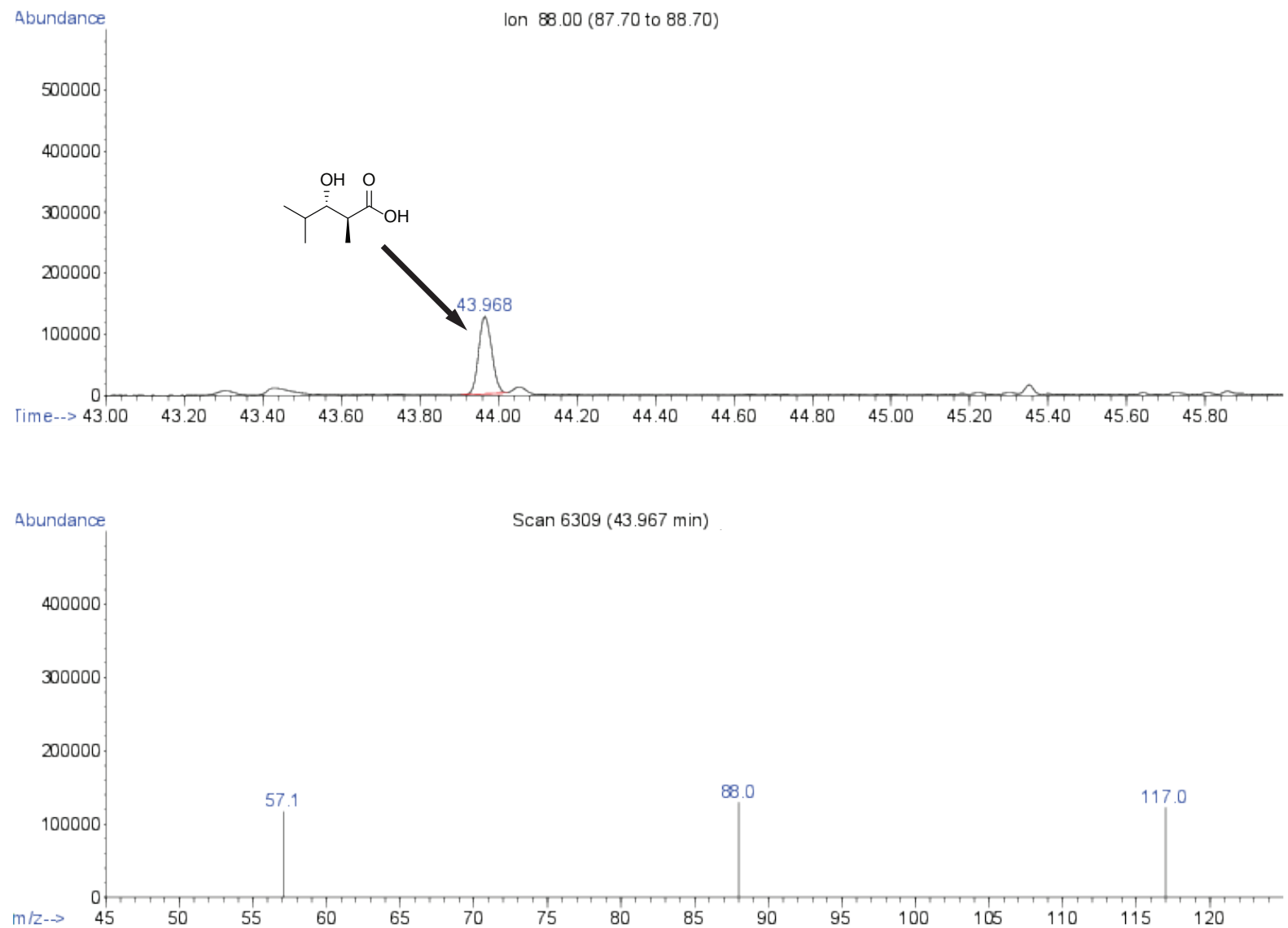

Figure 21: Stereochemical purity of 3a produced by (Amp KR $\mathrm{KR}_{1} \mathrm{LipPks}_{1}+\mathrm{TE}$. 

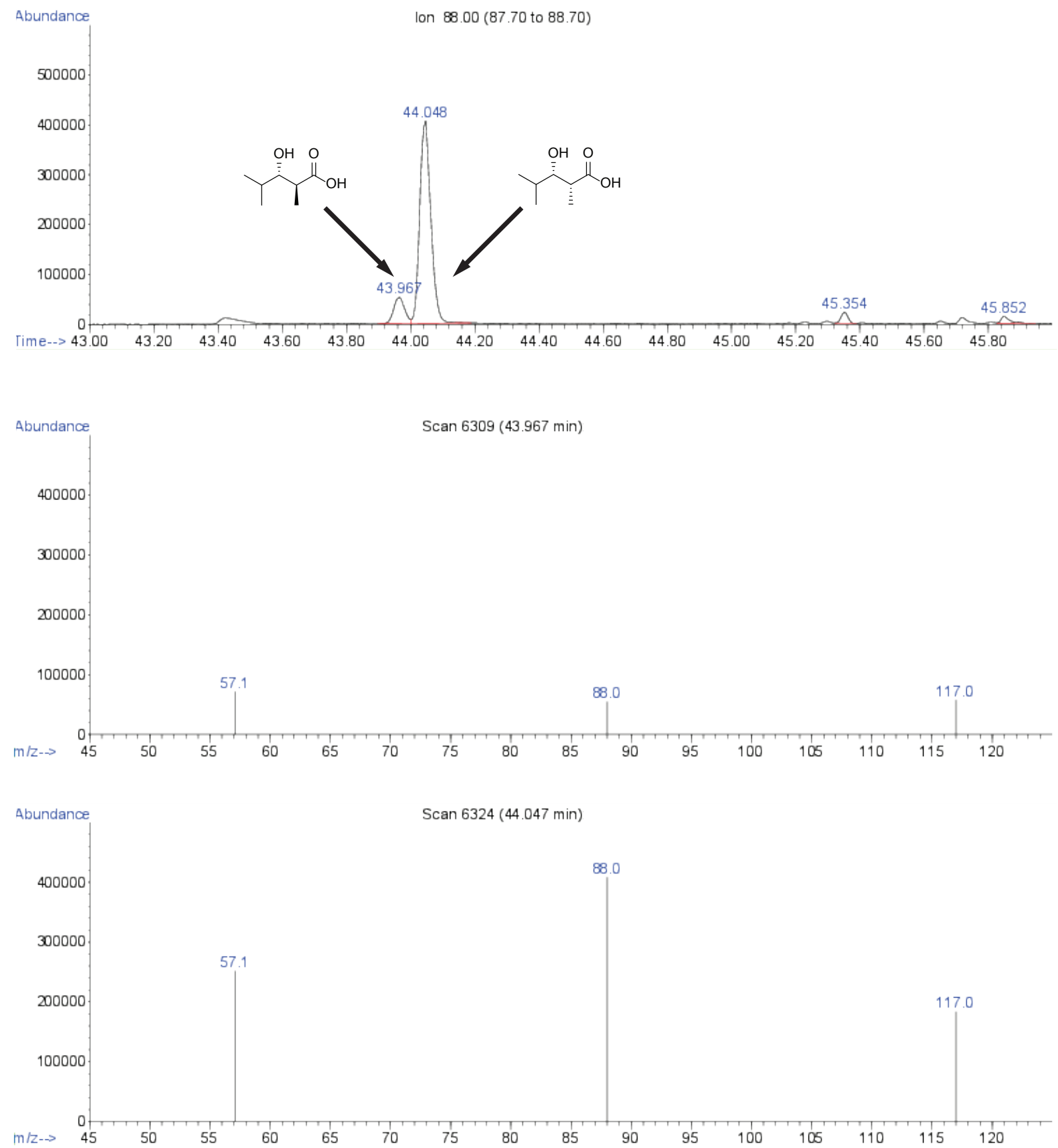

Figure 22: Stereochemical purity of $\mathbf{3 a}$ produced by $\left(\right.$ Ery $\left.\mathrm{KR}_{6}\right) \mathrm{LipPks} 1+\mathrm{TE}$. 


\section{Activity of LipPks1+TE Variants Using 1d as a Starter Sub- strate}

Table 5: Concentration of ketone $\mathbf{2 d}$ produced by LipPks1+TE variants after overnight incubation with substrate $\mathbf{1 d}$ in micromoles per liter, with n.d. used to indicate when no product was detected.

\begin{tabular}{|c|c|c|}
\hline \multirow{2}{*}{\multicolumn{2}{|c|}{$\begin{array}{c}\text { Substrate } \\
\text { Product }\end{array}$}} & $1 d$ \\
\hline & & $2 \mathrm{~d}$ \\
\hline Lip $K_{1}$ & $\mathrm{~A} 2$ & $33.6 \pm 4.1$ \\
\hline Amp $\mathrm{KR}_{2}$ & $\mathrm{~A} 1$ & $25.5 \pm 2.3$ \\
\hline $\operatorname{Amp} \mathrm{KR}_{2}{ }^{a}$ & $\mathrm{~A} 1$ & n.d. \\
\hline Con $\mathrm{KR}_{2}$ & B1 & $5.0 \pm 0.7$ \\
\hline Con $\mathrm{KR}_{2}{ }^{a}$ & B1 & n. \\
\hline Ery $\mathrm{KR}_{1}$ & $\mathrm{~B} 2$ & $18.0 \pm 2.3$ \\
\hline Bor $\mathrm{KR}_{1}$ & B & $6.5 \pm 0.3$ \\
\hline Spn $\mathrm{KR}_{3}$ & A1 & $37.4 \pm 3.3$ \\
\hline Amp $\mathrm{KR}_{1}$ & $\mathrm{~A} 2$ & $35.3 \pm 3.3$ \\
\hline Ery $\mathrm{KR}_{6}$ & 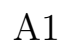 & $5.0 \pm 0.7$ \\
\hline
\end{tabular}

${ }^{a} \mathrm{DE}$ from the module furnishing the donor KR domain substituted for that of LipPks1.

Table 6: Stereochemistry of hydroxyacid 3d produced by A-type LipPks1+TE variants as evaluated by LC-MS, with n.d. used to indicate when no product was detected.

\begin{tabular}{|c|c|c|}
\hline \multicolumn{2}{|c|}{ Substrate } & $1 d$ \\
\hline \multicolumn{2}{|c|}{ Stereochemistry } & $\mathrm{A} 1 / \mathrm{B} 2 \mathrm{~A} 2 / \mathrm{B} 1$ \\
\hline Lip $K_{1}$ & A2 & $\bullet$ \\
\hline Amp $\mathrm{KR}_{2}$ & A1 & $\bullet$ \\
\hline $\operatorname{Amp~} \mathrm{KR}_{2}{ }^{a}$ & A1 & $\bullet$ \\
\hline Spn $\mathrm{KR}_{3}$ & A1 & $\bullet$ \\
\hline Amp $\mathrm{KR}_{1}$ & $\mathrm{~A} 2$ & n.d. \\
\hline Ery $\mathrm{KR}_{6}$ & A1 & $\bullet$ \\
\hline
\end{tabular}

${ }^{a} \mathrm{DE}$ from the module furnishing the donor KR domain substituted for that of LipPks1. 
14 Relative Production of 3a-d and 2a-d by LipPks1+TE Variants
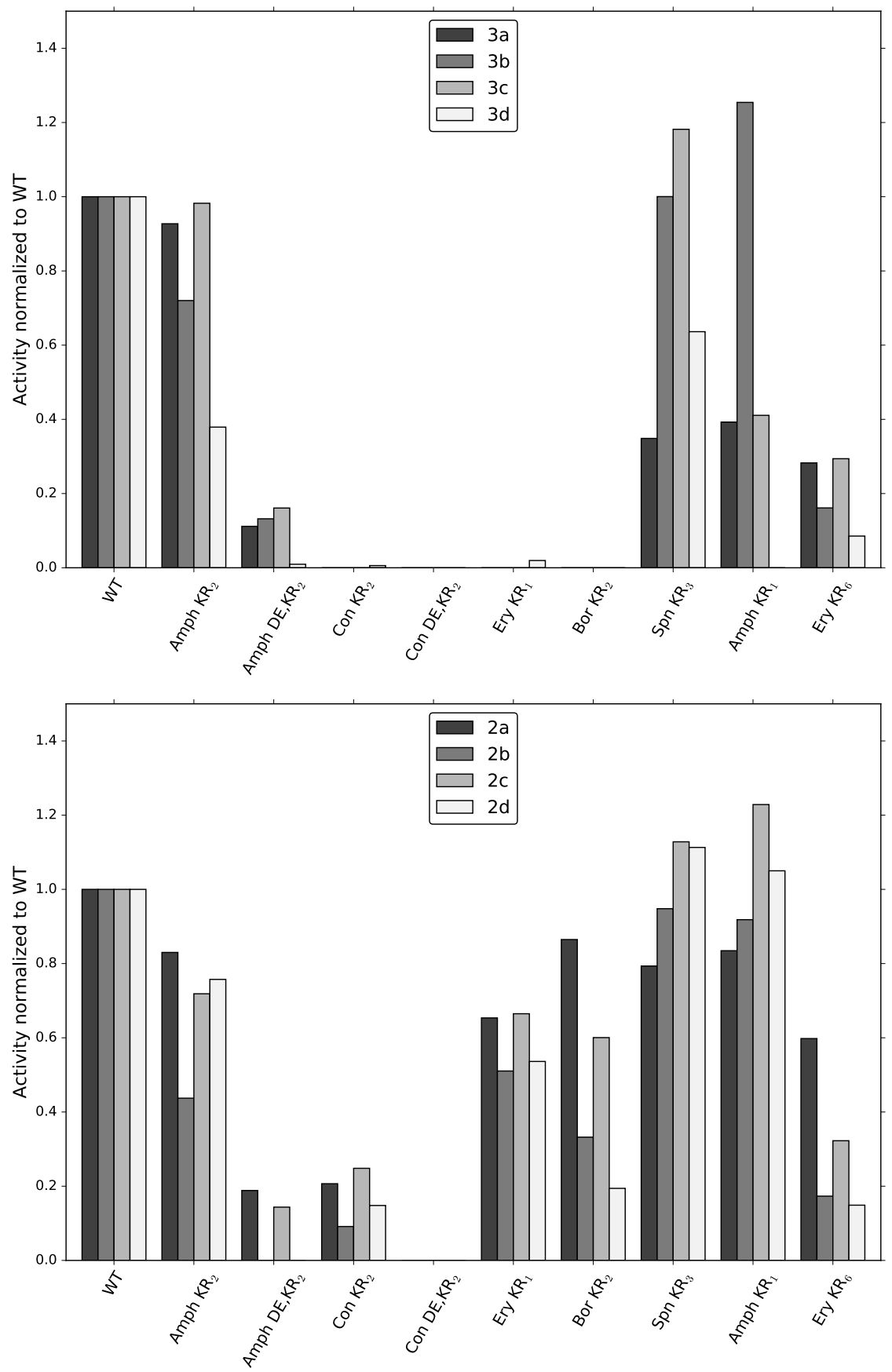

Figure 23: Production of hydroxyacids 3a-d (top) and ketones 2a-d (bottom) by LipPks1+TE variants normalized to production by wild-type LipPks1+TE. Production is calculated as the mean of experiments performed in triplicate. 


\section{References}

[1] V. Y. Alekseyev, C. W. Liu, D. E. Cane, J. D. Puglisi, and C. Khosla. Solution structure and proposed domain domain recognition interface of an acyl carrier protein domain from a modular polyketide synthase. Protein Sci., 16(10):2093-2107, 2007.

[2] C. Camacho, G. Coulouris, V. Avagyan, N. Ma, J. Papadopoulos, K. Bealer, and T. L. Madden. BLAST+: architecture and applications. BMC Bioinformatics, 10(1):421, 2009.

[3] K. R. Conway and C. N. Boddy. ClusterMine360: a database of microbial PKS/NRPS biosynthesis. Nucleic Acids Res., 41(Database issue):D402-D407, 2013.

[4] C. Engler, R. Gruetzner, R. Kandzia, and S. Marillonnet. Golden gate shuffling: a one-pot DNA shuffling method based on type IIs restriction enzymes. PLoS ONE, 4(5), 2009.

[5] C. Engler, R. Kandzia, and S. Marillonnet. A one pot one step precision cloning method with high throughput capability. PLoS ONE, 3(11), 2008.

[6] A. Hagen, S. Poust, T. de Rond, S. Yuzawa, L. Katz, P. D. Adams, C. J. Petzold, and J. D. Keasling. In vitro analysis of carboxyacyl substrate tolerance in the loading and first extension modules of borrelidin polyketide synthase. Biochemistry, 53(38):5975-5977, 2014.

[7] N. J. Hillson, R. D. Rosengarten, and J. D. Keasling. j5 DNA assembly design automation software. ACS Synth. Biol., 1(1):14-21, 2012.

[8] K. Katoh and D. M. Standley. MAFFT multiple sequence alignment software version 7: improvements in performance and usability. Mol. Biol. Evol., 30(4):772-780, 2013.

[9] A. T. Keatinge-Clay. A tylosin ketoreductase reveals how chirality is determined in polyketides. Chem. Biol., 14(8):898-908, 2007.

[10] A. T. Keatinge-Clay and R. M. Stroud. The structure of a ketoreductase determines the organization of the $\beta$-carbon processing enzymes of modular polyketide synthases. Structure, 14(4):737-748, 2006.

[11] S. Murli, J. Kennedy, L. C. Dayem, J. R. Carney, and J. T. Kealey. Metabolic engineering of Escherichia coli for improved 6-deoxyerythronolide B production. J. Ind. Microbiol. Biotechnol., 30(8):500-509, 2003.

[12] Y. Tang, C. Y. Kim, I. I. Mathews, D. E. Cane, and C. Khosla. The 2.7-A crystal structure of a 194-kDa homodimeric fragment of the 6-deoxyerythronolide B synthase. Proc. Natl Acad. Sci. U.S.A., 103(30):11124-11129, 2006.

[13] C. R. Valenzano, R. J. Lawson, A. Y. Chen, C. Khosla, and D. E. Cane. The biochemical basis for stereochemical control in polyketide biosynthesis. J. Am. Chem. Soc., 131(51):18501-18511, 2009 .

[14] S. Yuzawa, C. H. Eng, L. Katz, and J. D. Keasling. Broad substrate specificity of the loading didomain of the lipomycin polyketide synthase. Biochemistry, 52(22):3791-3793, 2013. 
[15] S. Yuzawa, L. Katz, and J. D. Keasling. Producing 3-Hydroxycarboxylic Acid and Ketone Using Polyketide Synthases. U.S. Patent Application 20150307855, Oct 29. 2015.

[16] J. Zheng and A. T. Keatinge-Clay. Structural and functional analysis of C2-type ketoreductases from modular polyketide synthases. J. Mol. Biol., 410(1):105-117, 2011.

[17] J. Zheng, S. K. Piasecki, and A. T. Keatinge-Clay. Structural studies of an A2-type modular polyketide synthase ketoreductase reveal features controlling $\alpha$-substituent stereochemistry. ACS Chem. Biol., 8(9):1964-1971, 2013.

[18] J. Zheng, C. A. Taylor, S. K. Piasecki, and A. T. Keatinge-Clay. Structural and functional analysis of A-type ketoreductases from the amphotericin modular polyketide synthase. Structure, 18(8):913-922, 2010. 